\title{
Fat tails arise endogenously from supply/demand, with or without jump processes
}

\author{
Gunduz Caginalp \\ University of Pittsburgh \\ Pittsburgh, PA 15260 \\ caginalp@pitt.edu
}

\begin{abstract}
We show that the quotient of Levy processes of jump-diffusion type has a fat-tailed distribution. An application is to price theory in economics, with the result that fat tails arise endogenously from modeling of price change based on an excess demand analysis resulting in a quotient of arbitrarily correlated demand and supply whether or not jump discontinuities are present. The assumption is that supply and demand are described by drift terms, Brownian (i.e., Gaussian) and compound Poisson jump processes. If $P^{-1} d P / d t$ (the relative price change in an interval $d t$ ) is given by a suitable function of relative excess demand, $(\mathcal{D}-\mathcal{S}) / \mathcal{S}$ (where $\mathcal{D}$ and $\mathcal{S}$ are demand and supply), then the distribution has tail behavior $F(x) \sim x^{-\zeta}$ for a power $\zeta$ that depends on the function $G$ in $P^{-1} d P / d t=G(\mathcal{D} / \mathcal{S})$. For $G(x) \sim|x|^{1 / q}$ one has $\zeta=q$. The empirical data for assets typically yields a value, $\zeta \cong 3$, or $\zeta \in[3,5]$ for many financial markets.

Many theoretical explanations have been offered for the disparity between the tail behavior of the standard asset price equation and empirical data. This issue never arises if one models price dynamics using basic economics methodology, i.e., generalized Walrasian adjustment, rather than the usual starting point for classical finance which assumes a normal distribution of price changes. The function $G$ is deterministic, and can be calibrated with a smaller data set. The results establish a simple link between the decay exponent of the density function and the price adjustment function, a feature that can improve methodology for risk assessment.

The mathematical results can be applied to other problems involving the relative difference or quotient of Levy processes of jump-diffusion type.
\end{abstract}

Key words. Quotient of Levy jump-diffusion processes, jump discontinuities, stochastic pricesasset price dynamics, supply and demand functions, fat tails.

1. Introduction. The calculation or approximation of a quotient of random variables or stochastic processes is considerably more important in mathematical modeling than it first appears. In many physical, biological and economics systems, there is a competition between two factors, e.g., supply and demand. The difference results in a temporal change in another important variable such as price. However, it is usually the relative difference that is relevant, and requires the division by one of the variables. Furthermore, if $X$ and $Y$ are the two random variables with some dimensions, and $Z$ is a dimensionless variable, and $\tau$ a time scale, then the equation $\tau d Z / d t=X-Y$ would not be meaningful from the dimensional analysis perspective. The right hand side must be divided by either $X, Y$ or a universal constant, or another variable with the same units. If $X$ and $Y$ are velocities (and relativistic), for example, the speed of light, $c$, would be a possible divisor. However, if there are no natural, universal constants, then it is likely from a dimensional analysis perspective that division by one of the variables is needed. This leads to consideration of an equation such as $\tau d Z / d t=(X-Y) / Y=X / Y-1$.

If $X$ and $Y$ are random variables, then the density of $\tau d Z / d t$ is given by the shifted density of the pure quotient $X / Y$. In particular, the density of a quotient has been studied in the literature for Gaussian random variables $X$ and $Y$ dating back to 1930 [28] (see references in [48]). The quotient has also been studied for a number of other distributions [56]. In most works, the random variable are assumed to be independent. The results are interesting in view of the broad range of approximate densities one obtains, 
including behavior that is similar to a Gaussian in the mid-range. Motivated by price changes in economics, Caginalp and Caginalp [7] studied the tail density problem for $X$ and $Y$ that are arbitrarily correlated Gaussian variables, finding an exact expression for the full density in the special case for which $X$ and $Y$ are anti-correlated. A closely related formula is proven in the Appendix A of this paper. In this paper we extend the general quotient results to include processes with jumps. Among the subtleties is the nature of correlations between the normal terms, the timing of the jumps and the magnitude of the jumps in the numerator and denominator.

For stochastic processes, $X_{t}$ and $Y_{t}$, one has similar questions. The mathematical problem we study in this paper is to determine the behavior of the tail of the distribution of $Z_{t}$ where (incorporating $\tau$ into the variable $Z$ ),

$$
Z_{t}=\frac{X_{t}}{Y_{t}}
$$

and $X_{t}$ and $Y_{t}$ are stochastic processes that consist of Brownian motion plus jump processes (more precisely, Levy processes of jump-diffusion type defined below).

The randomness in both $X_{t}$ and $Y_{t}$ is assumed to arise from the normals, the timing of the jumps, and the magnitude of the jumps.

Throughout this paper we focus on a classic price equation in economics, thereby addressing the problem through that perspective. However, the results are easily interpreted for other applications in physics, biology, etc.

A standard assumption in classical finance has been that relative changes in asset prices have a normal (Gaussian) distribution. In other words, with price $P(t)$ as a function of time, $t$, the price change $\Delta P(t):=$ $P(t+\Delta t)-P(t)$ in a time interval, $\Delta t$, satisfies $P^{-1} \Delta P \sim \mathcal{N}(\mu, \sigma \Delta t)$, i.e., a normal distribution with mean $\mu$ (the expected return) and variance $\sigma \Delta t$. This idea dates back to Bachelier's thesis [2] in 1900, and was broadly adopted by the finance community in mid-20th century for the pricing of options (e.g., Black-Scholes) and the assessments of risk. A popular idea for risk assessment has been the use of "valueat-risk" analysis 32 whereby one seeks to determine whether an investment will retain at least, say, $66 \%$ of its value with a probability of $90 \%$, for example, during the next decade. In addressing this question, classical methods use the historical data for that investment to determine the variance and the assumption of a normal distribution to calculate the value-at-risk probability.

Black-Scholes options pricing is also based on the idea that relative price change is normally distributed. In continuum form the basic stochastic equation is generally written as (see e.g., [2, 5, 32])

$$
P^{-1} d P=\mu d t+\sigma d W
$$

where $W$ is Brownian motion, so that $\Delta W:=W(t+\Delta t)-W(t) \sim \mathcal{N}(0, \Delta t)$ and $\mu$ and $\sigma$ are the mean and standard deviation of the stochastic process. The theoretical justification for equation (1) is limited, and its widespread use is partly attributable to mathematical convenience [11, 7] and the fact that it is a basic equation from which one can build additional models. In fact, one justification is based on infinite divisibility 1 . Roughly speaking, this means that the random price change in a particular time interval can be writen as a sum random variables on arbitrarily smaller time intervals. However, there are many other distributions besides Gaussian that are also infinitely divisible. So the property of infinite divisibility does not imply that one can apply the Central Limit Theorem to price changes.

A direct implication of the basic equation (11) is that the density of the relative price change on a fixed interval, $\Delta t$, is normal, e.g., for $\mu:=$ const, one has $P^{-1} \Delta P \sim \mathcal{N}\left(\mu, \sigma^{2} \Delta t\right)$ so that the density of the relative price change is

$$
f(x)=\left(2 \pi \sigma^{2} \Delta t\right)^{-1 / 2} \exp \left\{-(x-\mu)^{2} /\left(2 \sigma^{2} \Delta t\right)\right\},
$$

which exhibits the classical exponential decay for large $x$.

While there is some empirical justification for normality of relative price changes (often called return in the finance literature), the deviations between the data and a normal distribution become especially pronounced at the tails. This phenomenon of the tail of a density decaying at a rate that is a power law, i.e., much

\footnotetext{
${ }^{1}$ A probability distribution $F$ is infinitely divisible if for any integer $n \geq 2$ there exist independent, identically distributed random variables $X_{1}, . ., X_{n}$ such that $X_{1}+. .+X_{n}$ has distribution $F$. In particular, any Levy process, $\left\{X_{t}\right\}$ for any $t \geq 0$ has an infinitely divisible distribution ([12], p. 69).
} 
slower than the exponential of the normal distribution, is often called fat tails. A large discrepancy has been observed between the implications for the frequency of unusual events [51, 39, 47, 36, 23, 24, 13, 44, 57, 4, ?] in the data relative to that obtained from the normal distribution with parameters calculated from the standard deviation of a sample. Fat tails have also been observed in Bitcoin [16, Korea [17, and even in laboratory experiments [38] where uninformed traders are mainly responsible for these tails. Studies that have provided a theoretical framework for fat tails include [41, 44] [34, 43]. In particular, if one measures the standard deviation, $\sigma$, and the drift, or expected return, $\mu$, for the S\&P 500 and utilizes that value in the normal density above, then (11) would imply that a $4 \%$ drop, for example, occurs with a frequence that is one in many millions of days, instead of one in about 500 days, the observed frequency. Empirical data suggests that for most stock classifications, one has power law decay, i.e., $f(x) \sim x^{-\alpha}$ for some $\alpha>0$ (see Conclusion for comparison of our results with empirical data). The data for goods (see e.g., [37]) is less conclusive. The main objective of this paper is to prove that one obtains a power law decay from a price model that is based on economic principles of supply and demand (with any correlation) under conditions that include stochastic jumps in supply and demand.

The classical approach leading to (1) marginalizes the issues involving supply and demand, modeling instead the price change (11) as an empirically observed phenomenon. As an alternative to this semi-empirical equation (11), one can model price dynamics of assets directly through supply and demand, which ultimately drive price changes. As noted in [7 a simple and standard model for pricing of goods is the excess demand model that essentially describes price change in time as a mechanism to restore equilibrium (e.g. [59] or 60]). This is known in classical economics as Walrasian price adjustment, and is generally expressed as

$$
p_{t}-p_{t-1}=\frac{1}{\tau}\left(d_{t-1}-s_{t-1}\right),
$$

where $p_{t}$ is the price at the discrete time, $t$, and $s_{t-1}$ and $d_{t-1}$ are the supply and demand at time $t-1$, and $\tau^{-1}$ is a constant that determines the extent to which prices move for each unit of imbalance between supply and demand. The supply and demand functions are assumed to be linear, which are good approximations for smooth functions when deviations from the equilibrium point are small.

Of course, (2) is only a local equation that is valid for a particular pair of linear supply and demand near a point of intersection. For example, an imbalance created by $d_{t-1}=1,010$ and $s_{t-1}=1,000$ will have a much smaller impact on price change than would $d_{t-1}=20$ and $s_{t-1}=10$ even though $d_{t-1}-s_{t-1}=10$ in both cases. This demonstrates the need for normalization, realized by dividing the right hand side of (2) by $s_{t-1}$. Similarly, the left hand side must be normalized by dividing by $p_{t-1}$ leading to the equation, with $\tau_{0}$ now a dimensionless constant that characterizes the extent to which relative price changes as relative excess demand changes:

$$
\tau_{0} \frac{p_{t}-p_{t-1}}{p_{t-1}}=\frac{d_{t-1}-s_{t-1}}{s_{t-1}}
$$

The fact that we need to consider proportions of suply and demand implies that we have, instead of the difference $d_{t-1}-s_{t-1}$, the quotient minus 1, i.e., $d_{t-1} / s_{t-1}-1$. When we consider probability densities, the -1 simply produces a shift, so the probabilistic issues reduce to determining the density of a quotient of random variables.

Note that while these normalizations lead to an equation that is a reasonable non-local model, another feature of (22) is that it is a linear equation, so that the price change is always proportional to the excess demand. While linearity is often a convenient and reasonable approximation, there is no compelling requirement that price change be a linear function of excess demand. Introducing a differentiable function $g: \mathbb{R}^{+} \rightarrow \mathbb{R}$ with suitable properties 2 including $g(1)=0$ and $g^{\prime}>0$, we can write in place of (3) the equation

$$
\tau_{0} \frac{p_{t}-p_{t-1}}{p_{t-1}}=g\left(\frac{d_{t-1}}{s_{t-1}}\right) \text {. }
$$

\footnotetext{
${ }^{2}$ Note that we can write the generalization of the right hand side of (3) as

$$
\hat{g}\left(\frac{d_{t-1}-s_{t-1}}{s_{t-1}}\right)=\hat{g}\left(\frac{d_{t-1}}{s_{t-1}}-1\right)=g\left(\frac{d_{t-1}}{s_{t-1}}\right),
$$

so $g$ is defined as a shift of $\hat{g}$, i.e., $g(x)=\hat{g}(x-1)$.
} 
Thus, information on the supply and demand at any discrete time determines the price change for the next discrete time. The design of markets and efficient price discovery has been an active research area from both a theoretical and experimental perspective. See models by Milgrom [46, Gjerstad and Dickhaut [25], Hirshleifer et. al. 31], Gjerstad [27, [26, and references therein. The experimental aspect has been studied by researchers including Plott and Pogorelskiy, [49, Bossaerts and Plott, 4, Porter et. al. [50].

A modern approach to modeling price dynamics as asset flow, initiated in 1990, has been built on analyzing the supply and demand as discussed above (see e.g. [9, [6] and more recently [45, [15]). In other words, we build a continuum model based on the same principle as the discrete models above. The supply and demand can depend on the price, price derivative, and other factors.

There is little theoretical justification for assumption that relative price changes are normal, since the hypotheses for the Central Limit Theorem (CLT) do not apply directly to prices, as the latter evolve through a function of supply and demand. To be more specific, CLT involves the mean and variance of a large number of independent, identically distributed random variables. Prices are not in themselves independent random variables, but rather evolve through a process involving supply and demand. Moreover, supply and demand consist of a large number of independent agents placing buy and sell orders. Each buyer (and likewise, each seller) can be deemed to be a random variable, so that a large number of buyers can be reasonably approximated as independent and identically distributed (regardless of the particular distribution) so the case for applying the CLT is much stronger. See further discussion in Appendix D.

In this continuum formulation, the price equation can be written in its simplest form in terms of relative excess demand as

$$
\tau_{0} \frac{1}{P(t)} \frac{d P(t)}{d t}=\frac{\mathcal{D}(t)-\mathcal{S}(t)}{\mathcal{S}(t)}
$$

where supply, $\mathcal{S}$, and demand, $\mathcal{D}$, can vary due to any arbitrary set of motivations. For example, they can depend on the price, $P$, and the discount from an assessment of the true value of the asset. Here, $\tau_{0}$ is a time constant that also incorporates a constant rate factor that would multiply the right hand side. A key difference between the classical finance (11) and the asset flow approaches is due to fact that (11) assumes infinite arbitrage. The assumption is that there is always capital that can take advantage of mispricing of assets. In this way the deviation from realistic value will be small and random, and the random terms might be normal. In (5) the supply and demand can arise from various motivations and strategies including undervaluation and price trend. A derivation and discussion of this equation appear in [7]. See also modeling of supply/demand in [30, 31.

While the discrete price change mechanism may be more complicated that the models above indicate, one has to distinguish between a smoothly trading asset, such as a large capitialization stock, and an asset that trades in discrete quantities that cannot be approximated well by an equation such as (5). Thus, (4) may be a coarse approximation to a thinly traded asset, but a continuum limit such as (5) that arises from an averaging process is not likely to be sensitive to the infinitesimal perturbations of the supply and demand.

Note that in the price equation (5), supply and demand are not on an equal footing. This can be remedied by replacing the right hand side by a function $G(\mathcal{D} / \mathcal{S})$ with particular properties (see Section 2.2 of [8]), such as the function $G(u)=u-u^{-1}$. In other words, the simplest model, (5), may not be the one that corresponds to the empirical evidence for price change for a particular asset market. Thus, we assume the more general model

$$
\tau_{0} \frac{1}{P(t)} \frac{d P(t)}{d t}=G\left(\frac{\mathcal{D}(t)}{\mathcal{S}(t)}\right)
$$

with $G: \mathbb{R} \rightarrow \mathbb{R}$ a differentiable function 3 that satisfies such that $G^{\prime}>0$ and $G(1)=0$, and possibly other conditions.

We assume that price changes satisfy (15) where $\mathcal{D}$ and $\mathcal{S}$ are given by terms that include both Brownian process, $W(t)$, i.e., randomness with normal distribution, as well as jump discontinuities through a compound Poisson distribution.

We consider equation (5) for arbitrary correlation, $\rho$, between supply and demand, i.e., the most general case. There is some subtlety to the issue of correlation, since the correlation can arise from both the supply

\footnotetext{
${ }^{3}$ While differentiability is a desirable feature for studying dynamics, continuity suffices for the tail behavior results in this paper.
} 
and demand conditioned on the number of jumps or shock in each, as well as the correlation between the number of jumps (see Appendix C). The special case $\rho=-1$ (anti-correlation) involves the assumption that random terms that lead to higher demand also lead to lower supply with the same magnitude, and that the number of jumps is identical. In this limiting case one can use the exact density for a quotient using a modification of [7] in the Appendix A. We consider all cases, and prove that the following asymptotic relationship holds for each. For any $q>0$, the function obtained by smoothing [19] of the continuous function

$$
G(x)=\operatorname{sgn}\left(x-x^{-1}\right)\left|x-x^{-1}\right|^{1 / q},
$$

for example, corresponds to a distribution that decays as $F(x) \sim|x|^{-q}$. The values $q \in[3,5]$ corresponds the mid-range of the empirical results [24, 23], as discussed in the Conclusion.

While the results involve functions $G$ that are symmetric in supply and demand, one can similarly consider asymmetric functions (as determined by empirical data) whose properties will be manifested in the decay exponent analogously. In this paper we also consider the density conditioned on positive $\mathcal{D}$ and $\mathcal{S}$, in addition to unrestricted $\mathcal{D}$ and $\mathcal{S}$. The conditioning does not alter the tail exponent of the density.

In summary, a price dynamics equation based on excess demand yields a $q$ power law decay of the distribution function, whether or not there are jump discontinuities, complementing the earlier result [8] that a quotient of normals (without the Poisson jump terms) has a density with a power law decay. Furthermore, there is a simple link between the power, $q^{-1}$, in the deterministic price adjustment function, $G$, above, and decay exponent as indicated above.

There have been a variety of explanations for fat tails, such as the placement of large orders. Related to this is the perspective that fat tails are a consequence of jumps in prices. However, the placement of a large order has the direct effect of a jump in supply or demand; the change is price is a consequence. We model the changes in supply and demand that are responsible for changes in price and show that the tail of the distribution has the same power exponent with or without jump discontinuities.

Other approaches to modeling of prices and supply/demand and stochasticity include [33, [10]. Research has also provided simulations and theoretical models directly on prices (see, for example, [52], [3]).

From a mathematical perspective, the results obtained below are quite general. In many physical or biological models there are two processes that have Brownian motion and jump components (i.e., Levy processes of jump-diffusion type [12]; see also [55] for estimation and simulation using multi-variate Levy processes) that are in some type of competition, so the pertinent quantity is the difference between the two. However, as in the case of supply/demand, it is generally the relative difference that one needs to consider just to make the units balance. Thus, one has a quotient (with a shift of a constant, -1) of such Levy processes as a consequence. Hence, the analysis below applies to all such problems.

For maximum generality in a variety of applications, we consider quotients in which the numerator and denominator can be either (i) of either sign, or (ii) are conditioned to be positive. The issue of the sign of demand or supply in economics is considered in Appendix E.

2. The density of the quotient of diffusion-jump processes with arbitrary correlation and independent number of jumps. We consider stochastic processes consisting of Brownian plus a compound Poisson process (see Cont and Tankov [12] p. 111). Let $\mathcal{S}=X_{t}^{\mathcal{S}}, \mathcal{D}=X_{t}^{\mathcal{D}}$ be the stochastic supply and demand 4 at time $t$, with $\Delta X_{t}^{\mathcal{S}, \mathcal{D}}:=X_{t+\Delta t}^{\mathcal{S}, \mathcal{D}}-X_{t}^{\mathcal{S}, \mathcal{D}}$ for some finite interval, $\Delta t$. During a time interval $(t, t+\Delta t)$ one expects that the deterministic contribution to the supply and demand will be proportional to $\Delta t$, i.e., $\Delta X_{t}^{\mathcal{S}, \mathcal{D}}=\gamma_{\mathcal{S}, \mathcal{D}} \Delta t$ (where $\gamma_{\mathcal{S}}$ and $\gamma_{\mathcal{D}}$ are deterministic and independent of $\Delta t$ ) if there is no randomness. We let $D:=\gamma_{\mathcal{D}} \Delta t$ and $S:=\gamma_{\mathcal{S}} \Delta t$. Note that $\gamma_{S}$ and $\gamma_{D}$ can be regarded as the expected values of supply and demand (per unit time) and can depend on the asset's price and price trend, and other factors that would be coupled into the equations.

The stochastic supply and demand terms, $X_{t}^{\mathcal{S}}, X_{t}^{\mathcal{D}}$, incorporate a normal, i.e., Brownian motion, as well as a compound Poisson process. In addition to the deterministic term and Brownian motion, we allow for jumps whose timing is given by a Poisson process, while the jump distribution itself is normal. Let $\Delta N_{t}$ be the number of jumps in the demand during $(t, t+\Delta t)$, so that the probability that there are $k$ jumps in an

\footnotetext{
${ }^{4}$ Note that we assume that both the supply and demand are Levy jump diffusion processes, which, like a simple normal distribution, can be negative. This issue is discussed in the Appendix.
} 
interval $\Delta t$, is given by the Poisson distribution,

$$
\mathbb{P}\left(\left\{\Delta N_{t}=k\right\}\right)=e^{-\lambda_{1} \Delta t} \frac{\left(\lambda_{1} \Delta t\right)^{k}}{k !}
$$

and likewise for the supply, with $\tilde{N}_{t}$ similarly defined with parameter $\lambda_{2}$.

A key point here is that $N_{t}$ and $\tilde{N}_{t}$ are independent. However, the correlation between the numerator and denominator given $k_{1}$ jumps in the numerator and $k_{2}$ in the denominator will be a fixed $\rho \in(-1,1)$. In Section 5, we will consider a bivariate Poisson process whereby the number of jumps $\Delta N_{t}$ and $\Delta \tilde{N}_{t}$ in the demand and supply is arbitrarily correlated.

The jump processes in the numerator and denominator are defined by

$$
\sum_{i=N_{t}}^{N_{t+\Delta t}} Y_{i} \text { with } Y_{i} \sim \mathcal{N}\left(\mu_{1}, \sigma_{1}^{2}\right), \sum_{i=N_{t}}^{\tilde{N}_{t+\Delta t}} \tilde{Y}_{i} \text { with } \tilde{Y}_{i} \sim \mathcal{N}\left(\mu_{2}, \sigma_{2}^{2}\right) .
$$

Also, let $W_{t}$ and $\tilde{W}_{t}$ denote Brownian motion, $\mathcal{N}(0, \Delta t)$, for the demand and supply, respectively. Thus the ratic 5 of the change in supply and demand during $(t, t+\Delta t)$ is given by

$$
\frac{\Delta X_{t}^{\mathcal{D}}}{\Delta X_{t}^{\mathcal{S}}}=\frac{D\left(1+\frac{\sigma_{01}}{2} \Delta W_{t}+\sum_{i=N_{t}}^{N_{t+\Delta t}} Y_{i}\right)}{S\left(1+\frac{\sigma_{02}}{2} \Delta \tilde{W}_{t}+\sum_{i=N_{t}}^{\tilde{N}_{t+\Delta t}} \tilde{Y}_{i}\right)}
$$

The numerator and denominator are known as Levy processes of jump-diffusion type 12. We make the standard assumption that $\left\{\Delta W_{t}, Y_{i}\right\}$ are all mutually independent, as are $\left\{\Delta \tilde{W}_{t}, \tilde{Y}_{i}\right\}$. Note that we do not need to multiply the $Y_{i}$ sum by a coefficient since it can be incorporated into $\mu$ and $\sigma_{i}^{2}$. The correlation, $\rho$, between the random events entering the supply and demand will be specified below. Empirically, the supply and demand are generally negatively correlated, and often close to $\rho=-1$, however, we allow for the complete range $-1<\rho<1$. The case $\rho=-1$ will be considered in Section 4 through an exact expression for this special case of anti-correlation. The case $\rho=1$ is the trivial case in which the randomness in the supply and demand cancel, leaving only deterministic terms in (8). Further discussion of the correlation is presented in Appendix C, where we calculate the total correlation, $\rho_{T}$, between supply and demand and show that $\left|\rho_{T}\right|$ is bounded away from 1 when the jumps in the supply and demand are independent. In Section 6, we consider jumps that are not dependent and can consider the full range of $\rho_{T}$.

In the mathematical analysis below, it will be convenient to incorporate the deterministic terms, $D$ and $S$, into the constants as we consider the ratio $R_{a}:=R_{1} / R_{2}=\Delta X_{t}^{\mathcal{D}} / \Delta X_{t}^{\mathcal{S}}$. Thus, we write (noting that $\mu_{01}$ and $\mu_{02}$ will be proportional to $\Delta t$ )

$$
\begin{aligned}
& R_{1}:=\mu_{01}+\frac{\sigma_{01}}{2} \Delta W+\sum_{i=N_{t}}^{N_{t+\Delta t}} Y_{i} \\
& R_{2}:=\mu_{02}+\frac{\sigma_{02}}{2} \Delta \tilde{W}+\sum_{i=\tilde{N}_{t}}^{\tilde{N}_{t+\Delta t}} \tilde{Y}_{i} .
\end{aligned}
$$

Hence, the initial mathematical problems will be to determine the tail of the densities of $(i) R_{a}=R_{1} / R_{2}$; (ii) $R_{b}:=R_{a}-R_{a}^{-1}$, and $($ iii $) R_{c}:=s\left(R_{b}\right)$ where $s$ is an arbitrary function for the price dynamics equation.

Remark. In principle, all of the analysis below will be valid even if we had $\mu_{0 i}$ proportional to $(\Delta t)^{p}$ for $p>0$. In other words, obtaining the decay of the density is not contingent on having a meaningful limit as $\Delta t \rightarrow 0$.

An alternative that is used in Section 4 is to consider (8) without the $D / S$ (which is deterministic) factor, and then note that the exponent of the tail density is not altered upon multiplying the random variable by the deterministic factor $D / S$.

\footnotetext{
${ }^{5}$ The set of points on which the denominator vanishes has measure zero, and the finiteness of the integral representing the distribution is established in the sequel.
} 
We proceed by first determining the density of $R_{a}$ denoted $f_{a}$ conditioned on the number of jumps, $k_{i}$, for $R_{i}$, so that $N_{t}=k_{1}$ and $\tilde{N}_{t}=k_{2}$ are fixed. With this conditioning, we define a related variable where the $k_{i}$ are fixed:

$$
\begin{aligned}
& \hat{R}_{1}\left(k_{1}\right)=\mu_{01}+\frac{\sigma_{01}}{2} \Delta W+Y_{1}+\ldots+Y_{k_{1}} \\
& \hat{R}_{2}\left(k_{2}\right)=\mu_{02}+\frac{\sigma_{02}}{2} \Delta \tilde{W}+\tilde{Y}_{1}+\ldots+\tilde{Y}_{k_{2}} .
\end{aligned}
$$

Since the $Y_{i}$ are all independent, identically distributed variables (i.i.d.), and also independent of $\Delta W$, and likewise for $\tilde{Y}_{i}$, we have from (9) and (10))

$$
\begin{aligned}
& \hat{R}_{1}\left(k_{1}\right) \sim \mathcal{N}\left(\mu_{01}+k_{1} \mu_{1},\left(\frac{\sigma_{01}}{2}\right)^{2} \Delta t+k_{1} \sigma_{1}^{2} \Delta t\right), \\
& \hat{R}_{2}\left(k_{2}\right) \sim \mathcal{N}\left(\mu_{02}+k_{2} \mu_{2},\left(\frac{\sigma_{02}}{2}\right)^{2} \Delta t+k_{2} \sigma_{2}^{2} \Delta t\right) .
\end{aligned}
$$

We abbreviate $\sigma_{R_{1}}^{2}:=\left(\frac{\sigma_{01}}{2}\right)^{2}+k_{1} \sigma_{1}^{2} \Delta t, \quad \sigma_{R_{2}}^{2}:=\left(\frac{\sigma_{02}}{2}\right)^{2}+k_{2} \sigma_{2}^{2 \Delta t}, \mu_{R_{1}}:=\mu_{01}+k_{1} \mu_{1}, \mu_{R_{2}}:=\mu_{02}+k_{2} \mu_{2}$, yielding, $R_{i} \sim \mathcal{N}\left(\mu_{R_{i}}, \sigma_{R_{i}}\right), i=1,2$.

At this point we can assign an arbitrary correlation $\rho \in(-1,1)$ between the demand and supply (with $k_{1}$ and $k_{2}$ jumps or shocks) as follows. For each fixed pair $\left(k_{1}, k_{2}\right)$ one sets, for a given $\rho$,

$$
\operatorname{Cov}\left(\hat{R}_{1}\left(k_{1}\right), \hat{R}_{2}\left(k_{2}\right)\right)=\sigma_{12}\left(k_{1}, k_{2}\right):=\rho \sigma_{R_{1}}\left(k_{1}\right) \sigma_{R_{2}}\left(k_{1}\right) .
$$

Thus, $\rho$ is independent of $k_{1}$ and $k_{2}$, and has the prescribed value. Moreover, $\rho$ is an arbitrary parameter that can be determined empirically - as the correlation between the random events entering into the supply and demand.

Given two normals with prescribed means and variances, we can write down a joint density by specifying the correlation or covariance. In particular, the joint density of $R_{1}, R_{2}$ conditioned on $\Delta N_{t}=k_{1}$ and $\Delta \tilde{N}_{t}=k_{2}($ see 58 p. 7 ), is

$$
\begin{aligned}
f^{\left(k_{1}, k_{2}\right)}\left(x_{1}, x_{2}\right):= & f\left(x_{1}, x_{2} \mid \Delta N_{t}=k_{1}, \Delta \tilde{N}_{t}=k_{2}\right) \\
& =\frac{1}{2 \pi \sigma_{R_{1}} \sigma_{R_{2}}} \frac{1}{\sqrt{1-\rho^{2}}} \exp \left\{-\frac{1}{2\left(1-\rho^{2}\right)} Q\left(x_{1}, x_{2}\right)\right\} \\
Q\left(x_{1}, x_{2}\right) & :=\left(\frac{x_{1}-\mu_{R_{1}}}{\sigma_{R_{1}}}\right)^{2}-2 \rho\left(\frac{x_{1}-\mu_{R_{1}}}{\sigma_{R_{1}}}\right)\left(\frac{x_{2}-\mu_{R_{2}}}{\sigma_{R_{2}}}\right) \\
& +\left(\frac{x_{2}-\mu_{R_{2}}}{\sigma_{R_{2}}}\right)^{2} .
\end{aligned}
$$

The full density of $R_{1}$ and $R_{2}$ without the conditioning on $k_{i}$ is then

$$
\begin{gathered}
f\left(x_{1}, x_{2}\right)=\sum_{k_{1}, k_{2}=0}^{\infty} \mathbb{P}\left(\Delta N_{t}=k_{1}, \Delta \tilde{N}_{t}=k_{2}\right) \times \\
f\left(x_{1}, x_{2} \mid \Delta N_{t}=k_{1}, \Delta \tilde{N}_{t}=k_{2}\right) \\
f\left(x_{1}, x_{2}\right)=\sum_{k_{1}, k_{2}=0}^{\infty} e^{-\lambda_{1} \Delta t} \frac{\left(\lambda_{1} \Delta t\right)^{k_{1}}}{k_{1} !} e^{-\lambda_{2} \Delta t} \frac{\left(\lambda_{2} \Delta t\right)^{k_{2}}}{k_{2} !} \times \\
f\left(x_{1}, x_{2} \mid \Delta N_{t}=k_{1}, \Delta \tilde{N}_{t}=k_{2}\right) .
\end{gathered}
$$

Notation. For asymptotic relationships, we use the standard notation that for $g: \mathbb{R} \rightarrow \mathbb{R}$ we write for some $j \in \mathbb{N}$, the relations $g(x)=O\left(x^{-j}\right)$ or $g(x) \sim x^{-j}$ if there exist $c_{1}, c_{2}, x_{0} \in \mathbb{R}^{+}$such that

$$
c_{1} x^{-j} \leq g(x) \leq c_{2} x^{-j} \text { for all } x \geq x_{0} .
$$


We will use the notation $g(x) \simeq x^{p}$ if the difference $\left|g(x)-x^{p}\right|$ is bounded by a constant times the next power, $x^{p+1}$.

2.1a The density, $f_{a}$, of $\boldsymbol{R}_{a}$. With this joint density, $f\left(x_{1}, x_{2}\right)$, of $R_{1}$ and $R_{2}$ above, one can write the quotient density ([42] p. 187) as

$$
f_{R_{1} / R_{2}}(w)=\int_{-\infty}^{\infty}|y| f(w y, y) d y .
$$

For brevity let $c_{1}:=\left(2 \pi \sigma_{R_{1}} \sigma_{R_{2}} \sqrt{1-\rho^{2}}\right)^{-1}$ and $c_{2}:=\left(2\left(1-\rho^{2}\right)\right)^{-1}$ and write

$$
\begin{aligned}
f_{R_{1} / R_{2}}(w)= & c_{1} \int_{-\infty}^{\infty} d y|y| \sum_{k_{1}, k_{2}=0}^{\infty} e^{-\lambda_{1} \Delta t} \frac{\left(\lambda_{1} \Delta t\right)^{k_{1}}}{k_{1} !} e^{-\lambda_{2} \Delta t} \frac{\left(\lambda_{2} \Delta t\right)^{k_{2}}}{k_{2} !} \times \\
& \exp \left\{-c_{2}\left[\left(\frac{w y-\mu_{R_{1}}}{\sigma_{R_{1}}}\right)^{2}-2 \rho\left(\frac{w y-\mu_{R_{1}}}{\sigma_{R_{1}}}\right)\left(\frac{y-\mu_{R_{2}}}{\sigma_{R_{2}}}\right)+\left(\frac{y-\mu_{R_{2}}}{\sigma_{R_{2}}}\right)^{2}\right]\right\} .
\end{aligned}
$$

We denote

$$
I(w):=\int_{-\infty}^{\infty} d y|y| \exp \left\{-c_{2}\left[\left(\frac{w y-\mu_{R_{1}}}{\sigma_{R_{1}}}\right)^{2}-2 \rho\left(\frac{w y-\mu_{R_{1}}}{\sigma_{R_{1}}}\right)\left(\frac{y-\mu_{R_{2}}}{\sigma_{R_{2}}}\right)+\left(\frac{y-\mu_{R_{2}}}{\sigma_{R_{2}}}\right)^{2}\right]\right\}
$$

and approximate for large $|w|$. Let $z:=y-\frac{\mu_{R_{1}}}{w}$ so $h(z):=-\frac{c_{2}}{\sigma_{R_{1}}^{2}} z^{2}$ and

$$
\begin{aligned}
\left(\frac{w y-\mu_{R_{1}}}{\sigma_{R_{1}}}\right)\left(\frac{y-\mu_{R_{2}}}{\sigma_{R_{2}}}\right) & =\frac{w z\left(z+\frac{\mu_{R_{1}}}{w}-\mu_{R 2}\right)}{\sigma_{R_{1}} \sigma_{R_{2}}} \\
\left(\frac{y-\mu_{R_{2}}}{\sigma_{R_{2}}}\right)^{2} & =\frac{\left(z+\frac{\mu_{R_{1}}}{w}-\mu_{R 2}\right)^{2}}{\sigma_{R_{2}}^{2}}
\end{aligned}
$$

Using these identities we can write $I(w)$ as

$$
I(w)=\int_{-\infty}^{\infty} d z e^{w^{2} h(z)} g(z ; w)
$$

with

$$
g(z ; w):=\left|z+\frac{\mu_{R_{1}}}{w}\right| \exp \left\{2 c_{2} \rho \frac{w z\left(z+\frac{\mu_{R_{1}}}{w}-\mu_{R_{2}}\right)}{\sigma_{R_{1}} \sigma_{R_{2}}}-c_{2} \frac{\left(z+\frac{\mu_{R_{1}}}{w}-\mu_{R 2}\right)^{2}}{\sigma_{R_{2}}^{2}}\right\} .
$$

We can also write, using

$$
p_{k_{i}}:=e^{-\lambda_{i} \Delta t} \frac{\left(\lambda_{i} \Delta t\right)^{k_{i}}}{k_{i} !}
$$

the identity

$$
\begin{aligned}
f_{R_{1} / R_{2}}(w) & =\sum_{k_{1}, k_{2}=0}^{\infty} p_{k_{1}} p_{k_{2}} c_{1} \int_{-\infty}^{\infty} d z e^{w^{2} h(z)} g(z ; w) \\
& =\sum_{k_{1}, k_{2}=0}^{\infty} p_{k_{1}} p_{k_{2}} c_{1} I(w)
\end{aligned}
$$

The Laplace integral approximation ([18] p. 36) then yields

$$
\begin{aligned}
I(w) & \tilde{=} g(0 ; w)\left(\frac{-2 \pi}{w^{2} h^{\prime \prime}(0 ; w)}\right)^{1 / 2} e^{w^{2} h(0 ; w)} \\
& +e^{w^{2} h(0 ; w)} O\left(w^{-3}\right) .
\end{aligned}
$$


We compute $g(0 ; w)=\left|\frac{\mu_{R_{1}}}{w}\right| \exp \left\{-c_{2}\left(\frac{\mu_{R_{2}}}{\sigma_{R_{2}}}\right)^{2}\right\}, h(0)=0, h^{\prime \prime}(z)=-2 \frac{c_{2}}{\sigma_{2}^{2}}$. This yields

$$
I(w)=\left|\frac{\mu_{R_{1}}}{w}\right| \exp \left\{-c_{2}\left(\frac{\mu_{R_{2}}}{\sigma_{R_{2}}}\right)^{2}\right\} \pi^{1 / 2} \frac{\sigma_{R_{2}}}{c_{2}^{1 / 2}}\left|\frac{1}{w}\right| .
$$

Combining the constants in $c_{1} I(w)$ we have

$$
\pi^{1 / 2} \frac{c_{1}}{c_{2}^{1 / 2}} \mu_{R_{1}} \sigma_{R_{2}}=\left(\frac{\pi}{2}\right)^{1 / 2} \frac{\mu_{R_{1}}}{\sigma_{R_{1}}} .
$$

Thus, we can write the density of the quotient $R_{1} / R_{2}$ as (for large $|w|$ with the remainder term $O\left(|w|^{-3}\right)$ :

$$
\begin{aligned}
f_{R_{1} / R_{2}}(w) & \stackrel{=}{k_{k_{1}, k_{2}=0}^{\infty}} e^{-\lambda_{1} \Delta t} \frac{\left(\lambda_{1} \Delta t\right)^{k_{1}}}{k_{1} !} e^{-\lambda_{2} \Delta t} \frac{\left(\lambda_{2} \Delta t\right)^{k_{2}}}{k_{2} !} \\
& \times\left(\frac{\pi}{2}\right)^{1 / 2} \frac{\mu_{R_{1}}}{\sigma_{R_{1}}} \exp \left\{-c_{2}\left(\frac{\mu_{R_{2}}}{\sigma_{R_{2}}}\right)^{2}\right\} \frac{1}{|w|^{2}} .
\end{aligned}
$$

Remark. The convergence of this sum is clear. In particular, $\mu_{R_{i}}$ and $\sigma_{R_{i}}$ depend on $k_{1}$ and $k_{2}$ (and so does $c_{1}$ but not $c_{2}$ ), and one must consider these in terms of convergence of the infinite series. Recall

$$
\begin{aligned}
\mu_{R_{1}}:=\mu_{01}+k_{1} \mu_{1}, \quad \mu_{R_{2}}:=\mu_{02}+k_{2} \mu_{2} \\
\sigma_{R_{1}}^{2}:=\left(\frac{\sigma_{01}}{2}\right)^{2}+k_{1} \sigma_{1}^{2}, \quad \sigma_{R_{2}}^{2}:=\left(\frac{\sigma_{02}}{2}\right)^{2}+k_{2} \sigma_{2}^{2} .
\end{aligned}
$$

Thus, for large $k_{1}, k_{2}$, one has $\mu_{R_{1}} / \sigma_{R_{1}} \sim k_{1}^{1 / 2}$ and $\left(\mu_{R_{2}} / \sigma_{R_{2}}\right)^{2} \sim k_{2}$. Also, $\exp \left\{-c_{2}\left(\frac{\mu_{R_{2}}}{\sigma_{R_{2}}}\right)^{2}\right\}$ is less than 1. Thus, the end result is

$$
f_{R_{1} / R_{2}}(w) \sim \frac{e^{-\lambda_{1} \Delta t} e^{-\lambda_{2} \Delta t}}{|w|^{2}} \sum_{k_{1}, k_{2}=0}^{\infty} k_{1}^{1 / 2} \frac{\left(\lambda_{1} \Delta t\right)^{k_{1}}}{k_{1} !} \frac{\left(\lambda_{2} \Delta t\right)^{k_{2}}}{k_{2} !}<\infty .
$$

In other words, the factorials dominate the powers, and we have convergence for all values of the parameters.

2.2 The density $\boldsymbol{f}_{b}$ of $\boldsymbol{R}_{b}:=\boldsymbol{r}\left(\boldsymbol{R}_{a}\right):=\boldsymbol{R}_{a}-\boldsymbol{R}_{a}^{-1}$. Using the results above, we now focus on the behavior of the density (for large, positive values of the argument), $f_{b}$, for $R_{b}:=r\left(R_{a}\right)$, where $R_{a}:=R_{1} / R_{2}$ with density $f_{a}$.

The function $y=r(x):=x-x^{-1}$ has two smooth strictly monotonic branches (for $x>0$ and $x<0$ ) as shown in Figure 1. The respective inverses in the two regions are given by

$$
\begin{aligned}
& x_{ \pm}=h_{ \pm}(y)=\frac{1}{2}\left(y \pm \sqrt{y^{2}+4}\right), \\
& h_{ \pm}^{\prime}(y)=\frac{1}{2}\left(1 \pm\left(1+4 / y^{2}\right)^{-1 / 2}\right) .
\end{aligned}
$$

To calculate the density we first compute the large $|y|$ behavior for $h_{ \pm}(y)$ and its derivatives $h_{ \pm}^{\prime}(y)$. For a given positive value of $y$, the two intersections of $y$ with $r(x)$ will be denoted by $x_{+}^{>}$on the right half plane, and $x_{-}^{>}$on the left half plane. Both values are on the upper half plane as $y>0$. See Figure 1.

For $y \gg 1$ we have the approximations (with the error term being of the next power of $|y|$ )

$$
x_{+}^{>}=h_{+}(y) \simeq y, \quad x_{-}^{>}=h_{-}(y) \simeq \frac{1}{2}\left(1-\left(1+\frac{2}{y^{2}}\right)\right)=-\frac{1}{y} .
$$


In other words, when $y \gg 1$ one has either $x \gg 1$ (i.e., $x_{+}$on the right branch), or $-1 \ll x<0$ (i.e., $x_{-}$on the left branch) as shown by the intersections in Figure 1.

The derivatives for $y \gg 1$ are approximated by

$$
h_{+}^{\prime}(y) \simeq 1, \quad h_{-}^{\prime}(y) \simeq \frac{1}{y^{2}} .
$$

The analogous computations for $y \ll-1$ yield, with the two intersections $x_{ \pm}$on the right and left half planes (both in the lower half plane):

$$
\begin{aligned}
x_{+}^{<} & =h_{+}(y) \simeq \frac{1}{-y}, \quad x_{-}^{<}=h_{-}(y) \simeq y \\
h_{+}^{\prime}(y) & \simeq \frac{1}{y^{2}}, \quad h_{-}^{\prime}(y) \simeq 1 .
\end{aligned}
$$

In order to compute the density, $f_{b}(y)$, of $R_{b}$ we note that each value of $y$ for $R_{b}$ can be attained in two ways represented by the intersection of a constant value of $y$ with the two segments of $r(x)$. Thus, one has

$$
f_{b}(y)=f_{a}\left(h_{+}(y)\right) h_{+}^{\prime}(y)+f_{a}\left(h_{-}(y)\right) h_{-}^{\prime}(y) .
$$

For $y \gg 1$ we use the relations above in (22) and (23) together with the relation $f_{a}(y) \sim|y|^{-2}$ to obtain

$$
f_{b}(y) \sim f_{a}(y) \cdot 1+f_{a}\left(-\frac{1}{y}\right) \cdot \frac{1}{y^{2}} \sim \frac{1}{y^{2}} \quad \text { for } y \gg 1 .
$$

Note that we have used, for $y \gg 1$, the relation $f_{a}\left(-\frac{1}{y}\right) \simeq 1$.

Similarly, for $y \ll-1$ we have the relation

$$
f_{b}(y) \sim f_{a}\left(-\frac{1}{y}\right) \frac{1}{y^{2}}+f_{a}(y) \cdot 1 \sim \frac{1}{y^{2}} .
$$

Thus we can conclude that $f_{b}(y) \sim|y|^{-2}$ when $y \gg 1$.

2.3 The density for nonlinear functions in the price equation. We now consider a spectrum of nonlinear functions for the right hand side of the price adjustment equation, and prove that the tail of the density decays as a monomial, together with a calculation of the exponent. Recalling that $R_{a}$ represents the quotient of demand and supply, each modeled with a jump-diffusion process (i.e., Brownian motion together with a compound Poisson process), we consider specifically, for $q>0$, the random variable $R_{c}=G_{\varepsilon}\left(R_{a}\right)$ where $y=G_{\varepsilon}(x)$ is a smoothing (see [19]) of each branch of

$$
G(x)=\left\{\begin{array}{ccc}
\left(x-\frac{1}{x}\right)^{1 / q} & \text { if } & x-\frac{1}{x}>0 \\
-\left(\frac{1}{x}-x\right)^{1 / q} & \text { if } & x-\frac{1}{x}<0
\end{array} .\right.
$$

Note that the smoothing makes this differentiable at $x= \pm 1$. More general functions $G$ can be considered provided they satisfy the conditions $G(1)=0, G^{\prime}(x)>0$ for all $x \in \mathbb{R} \backslash\{ \pm 1,0\}$ and, if symmetry between $\mathcal{D}$ and $\mathcal{S}$ is imposed, $G(x)=-G\left(x^{-1}\right)$ (see [8]).

We calculate the decay in the density in two different ways each of which is useful for a general sets of functions replacing $G$.

2.3a Calculating $\boldsymbol{f}_{c}$ through $\boldsymbol{f}_{b}$. Given a positive real number $q$, we define a function $s: \mathbb{R} \rightarrow \mathbb{R}$ by

$$
s(u):=\left\{\begin{array}{ccc}
u^{1 / q} & \text { if } \quad u \geq 0 \\
-(-u)^{1 / q} & \text { if } \quad u<0
\end{array} .\right.
$$

and let $s_{\varepsilon}$ be the smoothing. Noting that $s_{\varepsilon}$ is strictly monotonic, with $y=s_{\varepsilon}(x)$ and inverse, $x=j_{\varepsilon}(y)$, which is a smoothing 6 of

$$
j(y)=\left\{\begin{array}{ccc}
y^{q} & \text { if } & y \geq 0 \\
-(-y)^{q} & \text { if } & y<0
\end{array} .\right.
$$

\footnotetext{
${ }^{6}$ The $j_{\varepsilon}$ will not be given by the same formula as $s_{\varepsilon}$, but this is not relevant for our purpose.
} 
Recalling $R_{b}=r\left(R_{a}\right)$ where $r(x):=x-x^{-1}$, the result $f_{b}(y) \sim|y|^{-2}$ for $|y| \gg 1$ and the density relation,

$$
f_{c}(y)=f_{b}\left(j_{\varepsilon}(y)\right) j_{\varepsilon}^{\prime}(y)
$$

we have for $y \gg 1$ the asymptotic relation,

$$
f_{c}(y) \sim f_{b}\left(y^{q}\right) y^{q-1} \sim y^{-q-1},
$$

and for $y \ll-1$ the same end result,

$$
f_{c}(y) \sim f_{b}\left(-\left(-y^{q}\right)\right) y^{q-1} \sim y^{-q-1} .
$$

2.3b Calculating $\boldsymbol{f}_{c}$ from $\boldsymbol{f}_{a}$ directly. We are interested first in $y=R_{c} \gg 1$, which can occur in two ways: $x \gg 1$, or $-1 \ll x<0$. We can write the inverse of $G_{\varepsilon}$, denoted $H$, in two continuous parts that lie in the $x>0$ and $x<0$ half-planes. The superscripts $>$ and $<$ denote, respectively, the parts expression of $H$ that are above and below the $x$ axis. Also, note that the regions $x-\frac{1}{x}>0$ correspond to $y>0$ for $y=G_{\varepsilon}(x)$ while $x-\frac{1}{x}<0$ corresponds $y<0$ in $y=G(x)$. The qualitative features for $G_{\varepsilon}(x)$ are similar to that for $r(x)$ above, and one can refer again to Figure 1.

In determining the density $f_{c}(y)$ for $y \gg 1$, we compute first the inverse of $y=G_{\varepsilon}(x)=\left(x-\frac{1}{x}\right)^{1 / q}$ in this region. Note that the roots of $x^{2}-y^{q} x-1=0$ are given by

$$
H_{ \pm}^{>}(y):=x_{ \pm}^{>}=\frac{y^{q} \pm \sqrt{y^{2 q}+4}}{2} .
$$

The positive root corresponds to $x \gg 1$ and is given by

$$
H_{+}^{>}(y)=x_{+}^{>}=\frac{y^{q}}{2}\left(1+\left(1+\frac{4}{y^{2 q}}\right)^{1 / 2}\right) \simeq \frac{y^{q}}{2}\left(1+\left(1+\frac{2}{y^{2 q}}\right)\right) \simeq y^{q} .
$$

The negative root corresponds to the left branch of $G$ so $H_{-}^{>}(y):=x_{-}^{>}$is given by

$$
\begin{aligned}
H_{-}^{>}(y) & :=x_{-}^{>}=\frac{y^{q}}{2}\left(1+\left(1+\frac{4}{y^{2 q}}\right)^{1 / 2}\right) \\
& \simeq \frac{y^{q}}{2}\left(1-\left(1+\frac{2}{y^{2 q}}\right)\right) \simeq-\frac{1}{y^{q}} .
\end{aligned}
$$

Computation of the derivatives yields

$$
\begin{aligned}
\frac{d}{d y} H_{ \pm}^{>}(y) & =\frac{1}{2}\left[q y^{q-1} \pm \frac{1}{2}\left(y^{2 q}+4\right)^{-1 / 2} 2 q y^{2 q-1}\right] \\
& =\frac{1}{2} q y^{q-1}\left(1 \pm \frac{y^{q}}{\left(y^{2 q}+4\right)^{1 / 2}}\right) ; \\
\frac{d}{d y} H_{+}^{>} & \sim y^{q-1} \text { and } \frac{d}{d y} H_{-}^{>}(y) \sim y^{-q-1}
\end{aligned}
$$

We can now compute the density as

$$
f_{c}(y)=f_{a}\left(H_{+}^{>}(y)\right) \frac{d}{d y} H_{+}^{>}(y)+f_{a}\left(H_{-}^{>}(y)\right) \frac{d}{d y} H_{-}^{>}(y),
$$

yielding the approximation

$$
f_{c}(y) \sim f_{a}\left(y^{q}\right) y^{q-1}+f_{a}\left(-\frac{1}{y^{q}}\right) y^{-q-1} .
$$

Recalling that $f_{a}\left(-y^{-q}\right) \stackrel{\sim}{=} f_{a}(0) \sim 1$, and $f_{a}(z) \sim z^{-2}$ we then have $f_{c}(y) \sim y^{-q-1}$. 
We consider next the case $y \ll-1$, which occurs in two ways: $0<x \ll 1$, or $x \ll-1$. These are the two intersections of a value of $y$ that is large and negative corresponding to the right and left branches, respectively, and the parts of $G_{\varepsilon}$ that lie below the $x$-axis.

On the negative parts of both branches we have the relation

$$
y=-\left(-x+\frac{1}{x}\right)^{1 / q}
$$

and thus $x^{2}+(-y)^{q}-1=0$, which has solutions

$$
\begin{aligned}
H_{ \pm}^{<}(y) & :=x_{ \pm}^{<}=\frac{-(-y)^{q} \pm \sqrt{(-y)^{2 q}+4}}{2} \\
& \simeq \frac{(-y)^{q}}{2}\left(-1 \pm\left(1+\frac{2}{(-y)^{2 q}}\right)\right) .
\end{aligned}
$$

We have then

$$
H_{+}^{<}(y) \sim \frac{1}{(-y)^{q}}, \quad H_{-}^{<} \sim-(-y)^{q} .
$$

The derivatives are given by

$$
\frac{d}{d y} H_{-}^{<}(y) \sim(-y)^{q-1}, \quad \frac{d}{d y} H_{+}^{<}(y) \sim(-y)^{-q-1} .
$$

For $y \ll-1$, we thereby compute the decay of the density as

$$
\begin{aligned}
f_{c}(y) & =f_{a}\left(H_{+}^{<}(y)\right) \frac{d}{d y} H_{+}^{<}(y)+f_{a}\left(H_{-}^{<}(y)\right) \frac{d}{d y} H_{-}^{<}(y) \\
& \sim f_{a}\left(\frac{1}{(-y)^{q}}\right)(-y)^{-q-1}+f_{a}\left(-(-y)^{q}\right)(-y)^{q-1} \\
& \sim 1 \cdot(-y)^{-q-1}+(-y)^{-2 q}(-y)^{q-1} \sim(-y)^{-q-1} .
\end{aligned}
$$

We have proven the following.

Theorem 2.3. Let $R_{a}:=R_{1} / R_{2}$ where $R_{1}=\mathcal{D}, R_{2}=\mathcal{S}$ are described by jump-diffusion processes (i.e., Brownian motion plus a compound Poisson process) through (9). Assume that the two Poisson processes, $\Delta N_{t}$ and $\Delta \tilde{N}_{t}$ are independent Poisson processes with arbitrary parameters $\lambda_{1}$ and $\lambda_{2}$. Let $\rho \in(-1,1)$ be the correlation between $R_{1}$ and $R_{2}$ conditioned on a fixed pair of jumps $\left(k_{1}, k_{2}\right)$. With $G_{\varepsilon}$ be defined via (26) , the density $f_{c}$ of $R_{c}:=G_{\varepsilon}\left(R_{a}\right)$ satisfies the asymptotic relation

$$
f_{c}(y) \sim|y|^{-q-1} \quad \text { for }|y| \gg 1 .
$$

3. The density functions conditioned on positivity. We consider the densities for functions of the ratio $R_{1} / R_{2}=\mathcal{D} / \mathcal{S}$ as before except that supply, $\mathcal{S}$, and demand, $\mathcal{D}$, are required to be positive random variables through conditioning.

3.1 The quotient with conditioning. The probability of $X / Y$ conditioned on $X>0$ and $Y>0$ is expressed as

$$
\mathbb{P}\left(\frac{X}{Y} \leq u \mid X>0, Y>0\right)=\frac{\mathbb{P}\left(\frac{X}{Y} \leq u, X>0, Y>0\right)}{\mathbb{P}(X>0, Y>0)} .
$$

Let $Q_{1}:=\mathbb{P}(X>0, Y>0)$ so that

$$
F_{X / Y}(u \mid X>0, Y>0)=Q_{1}^{-1} \underset{\substack{x / y \leq u, x>0, y>0}}{\int f_{X, Y}(x, y) d x d y}
$$


and using $w=x / y$ we have the conditional distribution and density as

$$
\begin{gathered}
F_{X / Y}(u \mid X>0, Y>0)=Q_{1}^{-1} \int_{0}^{\infty} d y \int_{0}^{u} y f_{X, Y}(w y, y) d w \\
f_{X / Y}(u \mid X>0, Y>0)=Q_{1}^{-1} \int_{0}^{\infty} y f_{X, Y}(u y, y) d y .
\end{gathered}
$$

In our case, the joint density $f_{X, Y}\left(x_{1}, x_{2}\right)$ is given by $f\left(x_{1}, x_{2}\right)$ in (14), (15) .

3.2 Calculation of $\boldsymbol{f}_{a}\left(\boldsymbol{y} \mid \boldsymbol{R}_{1}, \boldsymbol{R}_{2}>\mathbf{0}\right)$ for $|\boldsymbol{y}| \gg \mathbf{1}$. The density of the quotient $R_{a}:=R_{1} / R_{2}$ conditioned on $R_{1}, R_{2}>0$ is similar to the calculation in Section 2.1, with the main difference being that the integral $I(w)$ will involve only half of the interval, i.e., $\int_{0}^{\infty} y f_{X, Y}(w y, y) d y$, versus $\int_{-\infty}^{\infty}|y| f_{X, Y}(w y, y) d y$. The resulting asymptotics for large $|u|$ are the same (except for constant factors). Hence, we have the same asymptotic relation,

$$
f_{a}(w \mid X>0, Y>0) \sim|w|^{-2} \text { for }|w| \gg 1 .
$$

We use this to obtain results on the decay of the density of $R_{c}=G_{\varepsilon}(D / S)$ under the condition that supply, $\mathcal{S}$, and demand, $\mathcal{D}$ are both positive, utilizing two different approaches. In the first approach we find the conditional density of $R_{b}:=R_{a}-R_{a}^{-1}$ with $R_{a}:=R_{1} / R_{2}$, and then obtain the conditional density of $f_{c}$ from $f_{b}$ which is obtained from $f_{a}$. In the second approach we obtain it from $f_{a}$ directly.

3.3 Calculation of $\boldsymbol{f}_{c}\left(\boldsymbol{y} \mid \boldsymbol{R}_{1}, \boldsymbol{R}_{2}>\mathbf{0}\right)$ through $\boldsymbol{f}_{b}\left(\boldsymbol{y} \mid \boldsymbol{R}_{1}, \boldsymbol{R}_{2}>\mathbf{0}\right)$. Defining $R_{b}:=r\left(R_{a}\right)=$ $R_{a}-R_{a}^{-1}$, i.e., $y=r(x)=x-x^{-1}$ and conditioning on $R_{1}$ and $R_{2}$ positive, we have $R_{a}=R_{1} / R_{2}>0$, so only the right branch of $r(x)$, i.e., $x>0$, is of interest. It has inverse

$$
h_{+}(y):=x_{+}=\frac{1}{2}\left(y+\sqrt{y^{2}+4}\right) .
$$

As the conversion from $x$ to $y$ is single valued, the conditional density thereby satisfies, for both $y \gg 1$ and $y \ll-1$, the identity

$$
f_{b}\left(y \mid R_{1}, R_{2}>0\right)=f_{a}\left(h_{+}(y) \mid R_{1}, R_{2}>0\right) h_{+}^{\prime}(y) .
$$

Using the asymptotics obtained earlier, i.e.,(22), (24), we have for $y \gg 1$ (so $x \gg 1$ ) the asymptotic relation,

$$
f_{b}\left(y \mid R_{1}, R_{2}>0\right) \sim f_{a}(y) \cdot 1 \sim y^{-2} .
$$

and for $y \ll-1$ (so $-1 \ll x_{+}<0$ ) the result

$$
f_{b}\left(y \mid R_{1}, R_{2}>0\right) \sim f_{a}\left(\frac{1}{-y}\right) \frac{1}{y^{2}} \sim \frac{1}{y^{2}}
$$

Thus, we conclude that the density of $R_{b}$ conditioned on positive $R_{1}$ and $R_{2}$ yields the same asymptotic relation

$$
f_{b}\left(y \mid R_{1}, R_{2}>0\right) \sim y^{-2}, \quad|y|>>1 .
$$
of

Next, we use this result to calculate $f_{c}\left(y \mid R_{1}, R_{2}>0\right)$. We define $s_{\varepsilon}$ in the same way as the smoothing

$$
s(u):=\left\{\left(\begin{array}{ccc}
u^{1 / q} & \text { if } & u \geq 0 \\
-(-u)^{1 / q} & \text { if } & u<0
\end{array}\right) .\right.
$$

Note that $s_{\varepsilon}$ is strictly increasing and has an inverse $h_{\varepsilon}$ with $h_{\varepsilon}(y) \sim y^{q}$ if $|y| \gg 1$ (i.e., the smoothing does not alter the growth rate), and $h_{\varepsilon}^{\prime}(y) \sim 3 y^{2}$.

Thus, we have again, for $y \gg 1$

$$
\begin{aligned}
f_{c}\left(y \mid R_{1}, R_{2}>0\right) & =f_{b}\left(h_{\varepsilon}(y) \mid R_{1}, R_{2}>0\right) h_{\varepsilon}^{\prime}(y) \\
& \sim\left(y^{q}\right)^{-2} y^{q-1} .
\end{aligned}
$$


Similarly, we have for $y \ll-1$,

$$
\begin{aligned}
f_{c}\left(y \mid R_{1}, R_{2}>0\right) & =f_{b}\left(h_{\varepsilon}(y) \mid R_{1}, R_{2}>0\right) h_{\varepsilon}^{\prime}(y) \\
& \sim(-y)^{-2 q}(-y)^{q-1}=(-y)^{-q-1} .
\end{aligned}
$$

3.4 Calculation of $\boldsymbol{f}_{c}\left(\boldsymbol{y} \mid \boldsymbol{R}_{1}, \boldsymbol{R}_{2}>\mathbf{0}\right)$ through $\boldsymbol{f}_{a}\left(\boldsymbol{y} \mid \boldsymbol{R}_{1}, \boldsymbol{R}_{2}>\mathbf{0}\right)$ directly. We proceed in the same way as in Section $2.3 \mathrm{~b}$, where $R_{c}=G_{\varepsilon}\left(R_{a}\right)$ and $y=G_{\varepsilon}(x)$ is a smoothing of

$$
G(x)=\operatorname{sign}\left(x-x^{-1}\right)\left|x-x^{-1}\right|^{1 / q}, \quad q>0,
$$

Due to the conditioning on positive $R_{1}, R_{2}$, we have $R_{a}=R_{1} / R_{2}>0$, so we only need to consider the positive $x$ branch of $G_{\varepsilon}(x)$. The inverse $H_{+}(y)$ (with $H_{+}^{>}(y)$ for $y>0, H_{+}^{<}(y)$ for $y<0$ ) is defined in the same way as in Section $2.3 \mathrm{~b}$, and $H_{-}$is not relevant.

We have then for $y \gg 1$, the density relation (identical to Section $2.3 \mathrm{~b}$ except for the absence of $H_{-}$ term):

$$
f_{c}\left(y \mid R_{1}, R_{2}>0\right)=f_{a}\left(H_{+}^{>}(y) \mid R_{1}, R_{2}>0\right) \frac{d}{d y} H_{+}^{>}(y),
$$

yielding the approximation

$$
f_{c}\left(y \mid R_{1}, R_{2}>0\right) \sim f_{a}\left(y^{q} \mid R_{1}, R_{2}>0\right) y^{q-1}+f_{a}\left(-\frac{1}{y^{q}} \mid R_{1}, R_{2}>0\right) y^{-q-1} .
$$

Recalling that $f_{a}\left(-y^{-q} \mid \ldots\right) \simeq f_{a}(0) \sim 1$, and $f_{a}(z \mid \ldots) \sim z^{-2}$ we then have $f_{c}(y \mid \ldots) \sim y^{-q-1}$.

Similarly, for $y \ll-1$, we compute the decay of the density as

$$
\begin{aligned}
f_{c}\left(y \mid R_{1}, R_{2}>0\right) & =f_{a}\left(H_{+}^{<}(y) \mid \ldots\right) \frac{d}{d y} H_{+}^{<}(y) \\
& \sim f_{a}\left(\frac{1}{(-y)^{q}} \mid \ldots\right)(-y)^{-q-1} \sim(-y)^{-q-1} .
\end{aligned}
$$

Thus, we have the conclusion that for $|y| \gg 1$, the density satisfies the asymptotic relation

$$
f_{c}\left(y \mid R_{1}, R_{2}>0\right) \sim|y|^{-q-1} .
$$

We have then the following result.

Theorem 3.4. Let $R_{a}:=R_{1} / R_{2}$ where $R_{1}=\mathcal{D}, R_{2}=\mathcal{S}$ are described by jump-diffusion processes (i.e., Brownian motion plus a compound Poisson process) through (9). Assume that $\Delta N_{t}$ and $\Delta \tilde{N}_{t}$ are independent Poisson processes with arbitrary parameters $\lambda_{1}$ and $\lambda_{2}$. Let $\rho \in(-1,1)$ be the correlation between $R_{1}$ and $R_{2}$ conditioned on a fixed pair of jumps $\left(k_{1}, k_{2}\right)$. With $G_{\varepsilon}$ defined via (26), the density $f_{c}\left(y \mid R_{1}, R_{2}>0\right)$ of $R_{c}:=G_{\varepsilon}\left(R_{a}\right)$ conditioned on positive $R_{1}$ and $R_{2}$ satisfies the asymptotic relation

$$
f_{c}\left(y \mid R_{1}, R_{2}>0\right) \sim|y|^{-q-1} \quad \text { for }|y| \gg 1 .
$$

Remark. We conclude that the decay of the density $f_{c}$ is not altered by the conditioning on positive supply and demand.

4. The price function when supply and demand are anti-correlated $(\rho=-1)$. In this section, we consider the remaining case in which the supply and demand (i.e., $R_{1}$ and $R_{2}$ ) have correlation $\rho=-1$. For this particular case one can also derive an exact density from which one can analyze the $\Delta t \rightarrow 0$ limits.

4.1 The anti-correlated quotient of Levy processes of jump-diffusion type. We consider the quotient of demand, $\mathcal{D}=R_{1}$, and supply, $\mathcal{S}=R_{2}$ within a small time interval, now for the remaining case of $\rho=-1$, and write this quotient more simply as

$$
\frac{\mathcal{D}}{\mathcal{S}}=\frac{R_{1}}{R_{2}}=\frac{D\left(\Delta t+\frac{\sigma_{0}}{2} \Delta W_{t}+\sum_{i=N_{t}}^{N_{t+\Delta t}} Y_{i}\right)}{S\left(\Delta t-\frac{\sigma_{0}}{2} \Delta W_{t}-\sum_{i=N_{t}}^{N_{t+\Delta t}} Y_{i}\right)}
$$


We assume still that $\left\{\Delta W_{t}, Y_{i}\right\}$ are all mutually independent, with $\Delta W_{t} \sim \mathcal{N}(0, \Delta t)$ again, and write $Y_{i} \sim \mathcal{N}\left(\mu, \delta^{2}\right)$ to simplify notation.

The assumption of $\rho=-1$ in this limiting case implies that random events that lead to a rise in demand will simultaneously give rise to a fall in supply. Near the trading price anti-correlation is not far from a typical situation, since incoming news that is negative (for example due to an earnings downgrade) will cause a reassessment that impacts both the supply and demand with similar (though not exactly equal) magnitudes. In practice, they may have a strong negative correlation but greater than -1 .

Note that $S$ and $D$ are the deterministic factors in supply and demand (which we can regard as the expected values) while $\mathcal{S}$ and $\mathcal{D}$ are the complete supply and demand functions with stochasticity. We first define the quotient, $R_{a}$, without these factors:

$$
R_{a}:=\frac{\Delta t+\frac{\sigma_{0}}{2} \Delta W_{t}+\sum_{i=N_{t}}^{N_{t+\Delta t}} Y_{i}}{\Delta t-\frac{\sigma_{0}}{2} \Delta W_{t}-\sum_{i=N_{t}}^{N_{t+\Delta t}} Y_{i}}
$$

Since $Y_{i} \sim \mathcal{N}\left(\mu, \delta^{2}\right)$, if we examine $R_{a}$, conditioned on fixed $\Delta N_{t}=N_{t+\Delta t}-N_{t}=k$, we have a sum of $k+1$ normal variables plus a constant term $\Delta t$ in both the numerator and denominator, which thus have the respective normal distributions

$$
\mathcal{N}\left(\Delta t \pm k \mu, \frac{\sigma_{0}^{2}}{4} \Delta t+k \delta^{2}\right)
$$

An exact expression for a quotient of anti-correlated normal random variables, such as $R_{a}$, was derived in Theorem 4.3 of [7] under the condition of anti-correlation. For quotient of several other distributions, see [56]. If the numerator and denominator have distributions $\mathcal{N}\left(\mu_{1}, \sigma_{1}^{2}\right)$ and $\mathcal{N}\left(\mu_{2}, \sigma_{2}^{2}\right)$ respectively, then the quotient has density given by

$$
f(x)=\frac{\mu_{1} \sigma_{2}+\mu_{2} \sigma_{1}}{\sqrt{2 \pi}} \frac{\exp \left\{-\frac{1}{2}\left(\frac{\mu_{2} x-\mu_{1}}{\sigma_{2} x+\sigma_{1}}\right)^{2}\right\}}{\left(\sigma_{2} x+\sigma_{1}\right)^{2}} .
$$

Now let $\sigma_{1}^{2}=\sigma_{2}^{2}=\sigma^{2}:=\frac{\sigma_{0}^{2}}{4} \Delta t+k \delta^{2}, \mu_{1}:=\Delta t+k \mu$ and $\mu_{2}:=\Delta t-k \mu$ so that this general formula yields

$$
f_{a}\left(x \mid \Delta N_{t}=k\right)=\frac{2(\Delta t)}{\sqrt{2 \pi} \sigma} \frac{\exp \left\{-\frac{1}{2 \sigma^{2}}\left(\frac{(\Delta t)(x-1)-k \mu(x+1)}{x+1}\right)^{2}\right\}}{(x+1)^{2}} .
$$

Next, we define $R_{A}=\frac{D}{S} R_{a}$, which has density

$$
f_{A}\left(x \mid \Delta N_{t}=k\right)=\frac{f_{a}\left(\frac{x}{D / S} \mid \Delta N_{t}=k\right)}{D / S} .
$$

Substitution for $f_{a}$ yields

$$
f_{k}(x):=f_{A}\left(x \mid \Delta N_{t}=k\right)=\frac{2 \Delta t}{\sqrt{2 \pi} \sigma \frac{D}{S}} \frac{\exp \left\{-\frac{1}{2 \sigma^{2}}\left(\frac{(\Delta t)\left(\frac{x}{D / S}-1\right)-k \mu\left(\frac{x}{D / S}+1\right)}{\frac{x}{D / S}+1}\right)^{2}\right\}}{\left(\frac{x}{D / S}+1\right)^{2}} .
$$

5. Asymptotics of the density. We focus on the large $|x|$ behavior of the density by first examining (33) in the cases $k=0$ and $k \geq 1$ separately.

(i) For $k=0$, recalling $\sigma^{2}:=\frac{\sigma_{0}^{2}}{4} \Delta t+k \delta^{2}=\frac{\sigma_{0}^{2}}{4} \Delta t$, we have simply

$$
\begin{aligned}
f_{0}(x) & :=f_{A}\left(x \mid \Delta N_{t}=0\right) \\
& =\frac{1}{\sqrt{2 \pi} \frac{\sigma_{0}}{4(\Delta t)^{\frac{1}{2}}}} \frac{\frac{S}{D}}{\left(\frac{x}{D / S}+1\right)^{2}} \exp \left\{-\frac{1}{2} \frac{1}{\frac{\sigma_{0}^{2}}{4 \Delta t}}\left(\frac{\frac{x}{D / S}-1}{\frac{x}{D / S}+1}\right)^{2}\right\} .
\end{aligned}
$$


For $|x| \gg 1$ this yields the expression

$$
f_{0}(x) \sim \frac{1}{\sqrt{2 \pi} \frac{\sigma_{0}}{4(\Delta t)^{\frac{1}{2}}}} \frac{\frac{D}{S}}{x^{2}} \exp \left\{-\frac{1}{2} \frac{1}{\frac{\sigma_{0}^{2}}{4 \Delta t}}\right\} .
$$

(ii) For $k \geq 1$, and $|x| \gg 1$ we have similarly,

$$
f_{k}(x) \sim \frac{2(\Delta t) \frac{D}{S}}{\sqrt{2 \pi} \sigma} \frac{\exp \left\{-\frac{1}{2 \sigma^{2}}[\Delta t-k \mu]^{2}\right\}}{x^{2}},
$$

so the last two expressions both have a power law decay, $x^{-2}$.

Using the theorem of total probability, we now calculate the full density (i.e., without conditioning on $k$ )

$$
f_{A}(x)=\sum_{k=0}^{\infty} e^{-\lambda \Delta t} \frac{(\lambda \Delta t)^{k}}{k !} \frac{2 \Delta t}{\sqrt{2 \pi} \sigma \frac{D}{S}} \frac{\exp \left\{-\frac{1}{2 \sigma^{2}}\left(\frac{\mu_{2} \frac{x}{D / S}-\mu_{1}}{D / S}\right)^{2}\right\}}{\left(\frac{x}{D / S}+1\right)^{2}} .
$$

From the previously defined $f_{0}$, i.e., (34) and $f_{k}(k \geq 1)$, one has

$$
f_{A}(x)=e^{-\lambda \Delta t} f_{0}(x)+\sum_{k=1}^{\infty} e^{-\lambda \Delta t} \frac{(\lambda \Delta t)^{k}}{k !} f_{k}(x) .
$$

Considering the $k \geq 1$ terms separately, we have for $x \gg 1$,

$$
\sum_{k=1}^{\infty} e^{-\lambda \Delta t} \frac{(\lambda \Delta t)^{k}}{k !} f_{k}(x) \sim \sum_{k=1}^{\infty} e^{-\lambda \Delta t} \frac{(\lambda \Delta t)^{k}}{k !} \frac{2(\Delta t) \frac{D}{S}}{\sqrt{2 \pi} \sigma} \frac{\exp \left\{-\frac{1}{2 \sigma^{2}}[\Delta t-k \mu]^{2}\right\}}{x^{2}} .
$$

The series is clearly convergent so that the decay for large $|x|$ is $O\left(x^{-2}\right)$. Combining this with the similar result for $f_{0}(x)$ yields the conclusion

$$
f_{A}(x) \sim x^{-2} \quad \text { as } \quad|x| \rightarrow \infty .
$$

The results of Section 2 for $\rho \in(-1,1)$ follow similarly for the random variables $R_{b}:=R_{A}-R_{A}^{-1}$ and $R_{c}:=G_{\varepsilon}\left(R_{A}\right)$ where $G_{\varepsilon}$ is the smoothing of $G$ defined by (26) .

Theorem 5.1. Let $R_{A}$ be defined as the quotient of supply, $\mathcal{S}$, and demand, $\mathcal{D}$, through (30) with correlation $\rho=-1$. With $G_{\varepsilon}$ be defined via (26), the density $f_{c}$ of $R_{c}:=G_{\varepsilon}\left(R_{a}\right)$ satisfies the asymptotic relation

$$
f_{c}(y) \sim|y|^{-q-1}
$$

for $|y| \gg 1$.

The density of $R_{c}$ conditioned on the numerator and denominator both being positive also satisfies (41).

Proof. The last assertion follows from the exact conditional density for the quotient of anti-correlated normal random variables given in the Appendix A. Since this expression differs from (31) only by a constant, all of the asymptotic relationships remain valid in this conditional case. ///

Next, we consider the full density under the conditions $\Delta t \ll 1$ and $x \gg 1$. The $f_{0}(x)$ remains the same while $f_{k}(x)$ for $k \geq 1$ can be approximated using $\sigma^{2}=\frac{\sigma_{0}^{2}}{4} \Delta t+k \delta^{2} \tilde{=} k \delta^{2}$ to yield the following result for $\mu>0$. When $\mu=0$ with $\delta>0$, i.e., $Y_{i} \sim \mathcal{N}\left(0, \delta^{2}\right)$ we have no jumps in the Poisson process, so the randomness arises only through the Brownian motion which has been considered previously. 
Theorem 5.2 Under the same conditions as Theorem 5.1, but with $\Delta t \ll 1$ in addition to $x \gg 1$, and $\mu>0$, the density of $R_{A}$ is given by (38) with $f_{0}$ and $f_{k}$ having asymptotic behavior given by (35) and

$$
f_{k}(x) \sim \frac{2(\Delta t) \frac{D}{S}}{\sqrt{2 \pi} k^{1 / 2} \delta} \frac{\exp \left\{-\frac{1}{2} \frac{k \mu^{2}}{\delta^{2}}\right\}}{x^{2}}
$$

for $\mu>0$. Also, one has the bounds for sufficiently large $|x|$

$$
\begin{aligned}
\frac{2}{\sqrt{2 \pi}} \frac{\Delta t}{\delta} \frac{1}{x^{2}} \frac{D}{S} e^{-\lambda \Delta t} \frac{\lambda \Delta t e^{-\frac{\mu^{2}}{2 \delta^{2}}}}{2} & \leq \sum_{k=1}^{\infty} \frac{2(\Delta t) \frac{D}{S}}{\sqrt{2 \pi} k^{1 / 2} \delta} \frac{\exp \left\{-\frac{1}{2} \frac{k \mu^{2}}{\delta^{2}}\right\}}{x^{2}} \\
& \leq \frac{2}{\sqrt{2 \pi}} \frac{\Delta t}{\delta} \frac{1}{x^{2}} \frac{D}{S} e^{-\lambda \Delta t}\left[\exp \left(\lambda \Delta t e^{-\frac{\mu^{2}}{2 \delta^{2}}}\right)-1\right] .
\end{aligned}
$$

The proof is presented in Appendix B.

Remark. For nonlinear functions of $R_{A}$ one has similar expressions and decay using the methods of the previous sections.

Remark. Adding to (42) the $f_{0}(x)$ from (35), we see that asymptotic expression for density $f_{A}(x)$ (for $\Delta t \ll 1$ and $x \gg 1$ ) satisfies bounds obtained from (43) so that $f_{A}(x) \sim O\left(1 / x^{2}\right)$ for $x \gg 1$.

6. The density with arbitrary correlation between the number of jumps in the supply and demand. In this section we allow for the possibility that the number of jumps, $\Delta N_{t}$ in the demand (i.e., numerator) has an arbitrary correlation, $\rho_{J}$, with the supply (i.e., denominator). For positive reals $\lambda_{01}, \lambda_{02}, \lambda_{12}$ and $k, l \in \mathbb{N}$, we write the bivariate Poisson probability (see [35]) as

$$
p(k, l):=\mathbb{P}\left(\Delta N_{t}=k, \Delta \tilde{N}_{t}=l\right)=\sum_{j=0}^{\min \{k, l\}} \frac{\lambda_{01}^{k-j} \lambda_{02}^{l-j} \lambda_{12}^{j} e^{-\left(\lambda_{01}+\lambda_{02}+\lambda_{12}\right)}}{(k-j) !(l-j) ! j !}
$$

where $\mathbb{E}\left[\Delta N_{t}\right]=\operatorname{Var}\left[\Delta N_{t}\right]=\lambda_{01}+\lambda_{12}=: \lambda_{1}, \quad \mathbb{E}\left[\Delta \tilde{N}_{t}\right]=\operatorname{Var}\left[\Delta \tilde{N}_{t}\right]=\lambda_{02}+\lambda_{12}=: \lambda_{2}$. Summing over $l$ yields the individual probabilities,

$$
\mathbb{P}\left(\Delta N_{t}=k\right)=\frac{\left(\lambda_{01}+\lambda_{12}\right)^{k}}{k !} e^{-\left(\lambda_{01}+\lambda_{12}\right)},
$$

and similarly for $\mathbb{P}\left(\Delta \tilde{N}_{t}=l\right)$. Also, the covariance is given by $\operatorname{Cov}\left[\Delta N_{t}, \Delta \tilde{N}_{t}\right]=\lambda_{12}$ and, hence, the correlation by

$$
\rho^{(P)}:=\frac{\lambda_{12}}{\sqrt{\lambda_{01}+\lambda_{12}} \sqrt{\lambda_{02}+\lambda_{12}}} .
$$

Thus the correlation in the number of jumps can be adjusted through the parameters $\lambda_{01}, \lambda_{02}$, and $\lambda_{12}$. The analysis of Sections 2 and 3 can now be adapted for this general case by noting that the joint density for the random variables $\hat{R}_{i}\left(k_{i}\right)$ defined by (10) is still given by (13) and (14). At this point we do not assume independence of $\Delta N_{t}$ and $\Delta \tilde{N}_{t}$ but use the joint probability density above, and (15) is now replaced by

$$
f\left(x_{1}, x_{2}\right)=\sum_{k_{1}, k_{2}=0}^{\infty} p\left(k_{1}, k_{2}\right) f\left(x_{1}, x_{2} \mid \Delta N_{t}=k_{1}, \Delta \tilde{N}_{t}=k_{2}\right) .
$$

and similarly, (18) is identical except for the $p\left(k_{1}, k_{2}\right)$ substitution. The result (20) is then

$$
f_{R_{1} / R_{2}}(w) \stackrel{=}{=} \sum_{k_{1}, k_{2}=0}^{\infty} p\left(k_{1}, k_{2}\right)\left(\frac{\pi}{2}\right)^{1 / 2} \frac{\mu_{R_{1}}}{\sigma_{R_{1}}} \exp \left\{-c_{2}\left(\frac{\mu_{R_{2}}}{\sigma_{R_{2}}}\right)^{2}\right\} \frac{1}{|w|^{2}}
$$


One needs to show convergence of the sums above. Noting that $\mu_{R_{1}} / \sigma_{R_{1}} \sim k_{1}^{1 / 2}$ and the exponential is bounded by unity, one can prove the boundedness of the series by noting that

$$
\sum_{k_{1}, k_{2}=0}^{\infty} p\left(k_{1}, k_{2}\right) k_{1}^{1 / 2}<\sum_{k_{1}, k_{2}=0}^{\infty} p\left(k_{1}, k_{2}\right) k_{1}=\mathbb{E}\left[\Delta N_{t}\right]=\lambda_{01}+\lambda_{12}<\infty .
$$

Hence, we can state the conclusion analogous to Theorem 2.3. In addition, the analysis for $R_{1}$ and $R_{2}$ conditioned on positivity can be similarly carried out as in Section 3, with the following result.

Theorem 6.1. Let $R_{a}:=R_{1} / R_{2}$ where $R_{1}=\mathcal{D}, R_{2}=\mathcal{S}$ are described by jump-diffusion processes (i.e., Brownian motion plus a compound Poisson process) through (9). Assume that the two Poisson processes, $\Delta N_{t}$ and $\Delta \tilde{N}_{t}$ are bivariate Poisson with means $\lambda_{1}=\lambda_{01}+\lambda_{12}$ and $\lambda_{2}=\lambda_{02}+\lambda_{12}$ respectively, and covariance $\lambda_{12}$. Let $\rho \in(-1,1)$ be the correlation between $R_{1}$ and $R_{2}$ conditioned on a fixed pair of jumps $\left(k_{1}, k_{2}\right)$. With $G_{\varepsilon}$ be defined via (26) , the density $f_{c}$ of $R_{c}:=G_{\varepsilon}\left(R_{a}\right)$ satisfies the asymptotic relation

$$
f_{c}(y) \sim|y|^{-q-1} \text { for }|y| \gg 1 .
$$

The density $f_{c}\left(y \mid R_{1}, R_{2}>0\right)$ of $R_{c}:=G_{\varepsilon}\left(R_{a}\right)$ conditioned on positive $R_{1}$ and $R_{2}$ also satisfies the asymptotic relation

$$
f_{c}\left(y \mid R_{1}, R_{2}>0\right) \sim|y|^{-q-1} \text { for }|y| \gg 1 .
$$

The total correlation, $\rho_{T}$, as a function of the parameters is calculated in the Appendix C.

Summarizing the results, we see that the issue of correlation between supply and demand is complex. Given $k_{1}$ jumps or shocks for demand and $k_{2}$ for supply, we can impose a correlation, $\rho \in(-1,1)$, between supply and demand conditioned on $\left(k_{1}, k_{2}\right)$. However, the overall correlation (not conditioned on $k_{1}$ and $k_{2}$ ) for demand and supply will have a smaller magnitude if the number of jumps are independent, and is calculated in Appendix C.

In this section, we have considered the problem when the number of jumps in the supply and demand, $\Delta N_{t}$ and $\Delta \tilde{N}_{t}$ has an arbitrary correlation, $\rho^{(P)}$, given by (45) in which case one can obtain the full range $(-1,1)$ of correlation for the supply and demand, as stated in Theorem 6.1.

The limiting case in which the correlation between supply and demand is given by -1 , i.e., anti-correlation, has been considered in Theorem 5.1.

7. Conclusion. We have shown that fat tails can be obtained solely by modeling price adjustment through supply and demand, each of which are random variables consisting of Brownian motion and shocks from a Poisson distribution. This consistent with the empirical evidence for many asset markets. The classical theory embodied in (11) is based partly on empirical observations near the mean (and not the tails) of price changes. The fact that the normal distribution is a reasonable model near the mean - and is generally convenient mathematically - does not provide any evidence that the tail of density should behave as the normal does, i.e., $f(x) \sim e^{-x^{2} / 2}$. Prices evolve through re-balancing supply and demand. In this paper we have assumed a model that embodies the perspective of supply/demand without invoking the fine microstructure, and show that the quotient of two Levy processes of jump-diffusion type has a tail distribution that is a power law.

In essence, exponential decay in the classical finance models is a feature of the assumptions made without compelling theoretical arguments. Prices are not distributed by a random process; rather they are the consequence of changes in supply and demand which have randomness. In fact, it is almost a tautology to say that all price change is a direct consequence of changes in supply and demand, which have randomness. If the supply and demand were deterministic, there would be no randomness in price change. Aggregate supply and demand involve the sum of a large number of decision makers so that the Central Limit Theorem can be applied. The addition of a compound Poisson process to the randomness incorporates the influx of a smaller number of large orders that are manifested as jumps in supply or demand.

A natural way to model price change is through an excess demand model which involves a quotient of supply and demand. The important mathematical idea is that a quotient of two normals is not normal, 
though it resembles a normal near the mean for a range of parameters. The tail of the density of a quotient of normals has previously been shown to have a power law decay [7. In this paper, we showed that this persists even with the introduction of shocks in the form of a Poisson distribution when there is an arbitrary correlation in the number of shocks and an arbitrary total correlation between supply and demand.

Another important issue is that the price adjustment equation (5) is not necessarily linear. Hence, we consider appropriate nonlinear functions, $G_{\varepsilon}$, (26) that are characterized by an exponent $q^{-1}$ for $q>0$. The salient feature of this function is that supply and demand enter on equal footing.

For this family of functions we have calculated the power of the decay exponent for the density of the relative price change $P^{-1} \Delta P$, as $f(x) \sim x^{-q-1}$. The exponent is a function of the shape of the price function, $G_{\varepsilon}$, so that there is a direct link between the form of the price function and the decay exponent. Other possibilities for the function $G_{\varepsilon}$ for supply and demand yields similar results. Roughly speaking if the dependence of $G$ on the quotient of demand to supply is characterized by the power $q^{-1}$, then the decay will be $f(x) \sim|x|^{-\alpha}$ with $\alpha=1+q$.

Empirical studies of the tail of distributions for relative stock price changes (denoted by $r$ below) have mainly shown (see 23], 24], [53, [51, 44, 47] and references therein) that large capitalization stocks have a decay rate given by $\zeta \cong 3$ or at least within a range $\zeta \in[3,5]$ where

$$
P(\{|r|>x\}) \sim x^{-\zeta},
$$

so that $f(x) \sim x^{-\zeta-1}$. An early paper [29] studied 40 million data points and hypothesized a $\zeta=3$ "universal cubic law." Thus, we have $\alpha=q+1$, i.e., $\zeta=q$. This would predict that the $G_{\varepsilon}$ defined above should have $q^{-1}=1 / 3$ corresponding to $\alpha=4 \quad \zeta=3$. This means that if the nonlinear price function $G_{\varepsilon}$ has a behavior that is somewhat less responsive than linear, the implied decay will be within the empirical range. The analysis also suggests that it would be useful to do empirical research on determining the form of the function $G_{\varepsilon}$, as well as examining the correlation between the changes in supply and demand to determine how close it is to anti-correlation. Some studies (e.g., 40]) have indicated that the tail exponent varies to some extent as one examines different countries and markets such as emerging markets. While stock price movements have been studied extensively, there is much less empirical work on the nature of the supply and demand curves, particularly under extreme conditions. This would be a useful empirical work that could open up a new avenue to understand rare events as discussed below. This approach can be utilized not only for the tail but for the entire distribution so volatility can be modeled in its entirety.

Our model is applicable to any price formation, and is not restricted to financial instruments. In some markets for goods, the exponential tail may hold [37, 1]. Our analysis may provide a link between the tails of financial assets and goods/services. In our model, this would be attained by a function $G$ that is very slowly responding for large imbalance e.g., involving a logarithmic function. This would mean that as the supply/demand balance becomes extreme, the relative price does not continue to change nearly as much. It seems intuitively reasonable that the supply and demand curves for goods/services have this feature, since there is usually no possibility of resale. However, further research is needed on this issue.

In general, $G$ is a function to be calibrated. The essential point, however, is that $G$ is a deterministic function, while $\mathcal{D}$ and $\mathcal{S}$ are Levy processes of jump-diffusion type. To simplify the discussion, let us suppose that they are simply Gaussian. The central thesis is that price change is essentially deterministic given a specific supply and demand. Suppose that one is interested in estimating the probability of a $5 \%$ drop in an asset. In order to establish this probability directly from empirical data, one would need a very large amount of data since it occurs infrequently. However, using the formalism above, one would need many fewer observations that would determine the value of the price drop as a function of the imbalance in supply and demand. Once this connection is established, it is then a matter of understanding the probability of that imbalance. The supply, $\mathcal{S}$, and demand, $\mathcal{D}$, are each Gaussian, with means and variances that are easily estimated with a moderate amount of data (i.e., without necessarily observing rare events) so we know the density of the normalized excess demand, namely, $(\mathcal{D}-\mathcal{S}) / \mathcal{S}$.

In summary, we can establish the deterministic connection between the imbalance of supply/demand and the change in price through the function $G$. Once this is done, we can estimate the means and variances of the supply and demand individually. The mathematical results then yield the density of $(\mathcal{D}-\mathcal{S}) / \mathcal{S}$. With the approximation of $G$ we then obtain the probability of a particular size price drop. In particlar, we can estimate the distribution tail exponent, $\zeta=q$. 
Note that the use of a smaller data set is not just a matter of convenience. The issue is that as we use much larger sets, we are utilizing data that may be irrelevant, for example, because it is an artifact of a different era. Data sets of 252 trading days and 2520 correspond to one year and ten years, respectively. In the year 2011, for example, the ten year period would include the post-Internet bubble of 2001-2003, as well as the 2007-2008 housing debacle and subsequent recovery. So data from only the period 2010-2011 would be more pertinent in terms of estimating the probabilities of possible rare events in 2012. Then using the data from 252 or 504 relevent trading days, one can find the most extreme price changes and match them up with the $(\mathcal{D}-\mathcal{S}) / \mathcal{S}$ values, so that the values of $G$ for those arguments are set. This is a consequence of $G$ being deterministic. From this data set of 252 or 504 we can reliably determine the mean and variance of each of supply and demand, noting that these are Gaussian by virtue of the Central Limit Theorem for independent agents. Using these parameters, and the analysis of the quotient of Levy jump-diffusion processes, we can calculate a more accurate probabilty for the extreme events that were observed than would be possible by using just the raw frequency.

The problem of fat tails is part of the general issue of volatility in markets that has been of great interest in recent years (see, e.g., [22]). The methods discussed can be useful within the general setting of volatility problems.

The analysis of this paper has involved normal distributions through the Brownian motion and the distribution of each jump in the Poisson process. An open problem is to extend these results to a broad set of distributions. Using results such as [56, one can consider the quotient other distributions for the supply and demand and analyze the tail behavior.

Appendix A. The density of a quotient of anti-correlated normal random variables with positive numerator and denominator.

We obtain a result that is a modification of Theorem 4.3 of [7. We define, for $\mu_{1}, \sigma_{1}, \mu_{2}, \sigma_{2}>0$ and $Z \sim \mathcal{N}(0,1)$

$$
R:=\frac{\mu_{1}+\sigma_{1} Z}{\mu_{2}-\sigma_{2} Z}
$$

and wish to calculate (neglecting sets of measure zero)

$$
P_{+}:=\mathbb{P}\left\{R \leq x \mid \mu_{1}+\sigma_{1} Z>0, \mu_{2}-\sigma_{2} Z>0\right\}=\frac{\mathbb{P}\left\{R \leq x, \mu_{1}+\sigma_{1} Z, \mu_{2}-\sigma_{2} Z>0\right\}}{\mathbb{P}\left\{\mu_{1}+\sigma_{1} Z>0, \mu_{1}-\sigma_{1} Z>0\right\}}
$$

Let $Q:=\mathbb{P}\left\{\mu_{1}+\sigma_{1} Z>0, \mu_{1}-\sigma_{1} Z>0\right\}$ so that $Q=\int_{-\mu_{1} / \sigma_{1}}^{\mu_{2} / \sigma_{2}}(2 \pi)^{-1 / 2} e^{-x^{2} / 2} d x$. We have then

$$
Q P_{+}=\mathbb{P}\left\{\mu_{1}+\sigma_{1} Z \leq\left(\mu_{2}-\sigma_{2} Z\right) x, \mu_{1}+\sigma_{1} Z>0, \mu_{1}-\sigma_{1} Z>0\right\} .
$$

The three inequalities above reduce to (i) $Z \leq \frac{\mu_{2} x-\mu_{1}}{\sigma_{2} x+\sigma_{1}}$, (ii) $Z \geq-\mu_{1} / \sigma_{1}$, and (iii) $Z<\mu_{2} / \sigma_{2}$. Since the inequality $\left(\right.$ iii) is less restrictive than $(i)$, we can ignore (iii) and write, with $f_{Z}(s)=(2 \pi)^{-1 / 2} e^{-x^{2} / 2}$ as the density for $Z \sim \mathcal{N}(0,1)$ :

$$
Q P_{+}=\int_{-\mu_{1} / \sigma_{1}}^{\frac{\mu_{2} x-\mu_{1}}{\sigma_{2} x+\sigma_{1}}} f_{Z}(s) d s .
$$

Differentiating this expression with respect to $x$ yields the result that we can express in the form

$$
f_{X / Y}(x \mid X>0, Y>0)=\frac{\mu_{1} \sigma_{2}+\mu_{2} \sigma_{1}}{\sqrt{2 \pi} Q} \frac{e^{-\frac{1}{2}\left(\frac{\mu_{2} x-\mu_{1}}{\sigma_{2} x+\sigma_{1}}\right)}}{\left(\sigma_{2} x+\sigma_{1}\right)^{2}} .
$$

Appendix B. Proof of Theorem 5.2. We examine bounds on these asymptotic expressions for the full density if both $\Delta t \ll 1$ and $x \gg 1$ [i.e.,(38) with (35) and (42)] and obtain

$$
\begin{aligned}
\sum_{k=1}^{\infty} f_{k}(x) e^{-\lambda \Delta t} \frac{(\lambda \Delta t)^{k}}{k !} & \sim \frac{2}{\sqrt{2 \pi}} \frac{\Delta t}{\delta} \frac{D}{S} \frac{1}{x^{2}} e^{-\lambda \Delta t} \sum_{k=1}^{\infty} \frac{(\lambda \Delta t)^{k} \exp \left\{-\frac{1}{2} \frac{k \mu^{2}}{\delta^{2}}\right\}}{k^{1 / 2} k !} \\
& =C A,
\end{aligned}
$$




$$
C:=\frac{2}{\sqrt{2 \pi}} \frac{\Delta t}{\delta} \frac{D}{S} \frac{1}{x^{2}} e^{-\lambda \Delta t}, \quad A:=\sum_{k=1}^{\infty} \frac{(\lambda \Delta t)^{k} \exp \left\{-\frac{1}{2} \frac{k \mu^{2}}{\delta^{2}}\right\}}{k^{1 / 2} k !} .
$$

Upon defining

$$
A_{1}:=\sum_{k=1}^{\infty} \frac{(\lambda \Delta t)^{k} \exp \left\{-\frac{1}{2} \frac{k \mu^{2}}{\delta^{2}}\right\}}{k !}, \quad A_{2}:=\sum_{k=1}^{\infty} \frac{(\lambda \Delta t)^{k} \exp \left\{-\frac{1}{2} \frac{k \mu^{2}}{\delta^{2}}\right\}}{(k+1) !}
$$

we have

$$
A_{2} \leq A \leq A_{1} .
$$

We let $a:=\lambda \Delta t e^{-\frac{1}{2} \frac{\mu^{2}}{\delta^{2}}}$ so

$$
\begin{aligned}
& A_{1}=e^{a}-1 \\
& A_{2}=\frac{1}{a}\left(e^{a}-1-a\right) \geq a / 2 .
\end{aligned}
$$

satisfy the bounds

$$
\begin{gathered}
C A_{2} \leq C A \leq C A_{1} . \\
C \frac{a}{2} \leq C \frac{1}{a}\left(e^{a}-1-a\right)=C A_{2} \leq C A \leq C A_{1} \leq C\left(e^{a}-1\right), \text { i.e. }
\end{gathered}
$$

yielding (43).

Noting also that the $f_{0}(x)$ term cannot be simplified further beyond (35) for $\Delta t \ll 1$ we see from (38) that all terms decay as $x^{-2}$ for $x>>1$.

Appendix C. Analysis of supply/demand correlation. In Section 2 we considered an arbitrary correlation, $\rho$, between supply and demand when there are $k_{1}$ jumps or shocks in demand, and $k_{2}$ in supply during a small time interval, $\Delta t$, determined by independent Poisson processes, though the magnitude of the jumps in supply and demand are correlated. The independence of the Poisson processes implies an upper bound to the magnitude of the correlation between the supply and demand. In Section 6 we utilized a bivariate Poisson distribution for the jumps, which means that the full range $\rho \in(-1,1)$ can be attained. Both of these assertions will be established, together with an exact calculation in this Appendix.

We start with $R_{1}$ and $R_{2}$ defined as earlier by

$$
R_{1}=\mu_{01}+\frac{\sigma_{01}}{2} \Delta W+\sum_{j=0}^{\Delta N_{t}} Y_{j}, \quad R_{2}=\mu_{02}+\frac{\sigma_{02}}{2} \Delta \tilde{W}+\sum_{j=0}^{\Delta N_{t}} \tilde{Y}_{j}
$$

where $Y \sim \mathcal{N}\left(0, \sigma_{1}\right)$ and $\tilde{Y} \sim \mathcal{N}\left(0, \sigma_{2}\right)$, and we have set $\mu_{1}=\mu_{2}=0$ so that the drift arises only from the first term, implying $\mathbb{E} R_{1}=\mu_{01}, \mathbb{E} R_{2}=\mu_{02}$. The variables $\hat{R}_{1}\left(k_{1}\right)$ and $\hat{R}_{2}\left(k_{2}\right)$ are the corresponding values when $\Delta N_{t}=k_{1}$ and $\Delta \tilde{N}_{t}=k_{2}$, namely, (10).

We calculate, using the notation $\mathbb{P}\left\{\Delta N_{t}=k_{1}, \Delta \tilde{N}_{t}=k_{2}\right\}=: p\left(k_{1}, k_{2}\right)$, the identity,

$$
\begin{aligned}
\operatorname{Var} R_{1} & =\mathbb{E}\left[\left(R_{1}-\mathbb{E} R_{1}\right)^{2}\right]=\mathbb{E}\left[\left(\frac{\sigma_{01}}{2} \Delta W+\sum_{j=0}^{\Delta N_{t}} Y_{j}\right)^{2}\right] \\
& =\sum_{k_{1}, k_{2}=0}^{\infty} \mathbb{E}\left[\left(\frac{\sigma_{01}}{2} \Delta W+\sum_{j=0}^{\Delta N_{t}} Y_{j}\right)^{2} \mid \Delta N_{t}=k_{1}, \Delta \tilde{N}_{t}=k_{2}\right] p\left(k_{1}, k_{2}\right) \\
& =\sum_{k_{1}, k_{2}=0}^{\infty} \mathbb{E}\left[\left(\frac{\sigma_{01}}{2} \Delta W+\sum_{j=0}^{k_{1}} Y_{j}\right)^{2}\right] p\left(k_{1}, k_{2}\right) .
\end{aligned}
$$


Since the $\left\{Y_{j}\right\}$ are independent of each other and $\Delta W$, we have

$$
\operatorname{Var} R_{1}=\sum_{k_{1}, k_{2}=0}^{\infty} p\left(k_{1}, k_{2}\right)\left(\left(\frac{\sigma_{01}}{2}\right)^{2}+k_{1} \sigma_{1}^{2}\right)
$$

Now using the fact that the mean and variance of $p_{k_{1}}$, defined in (7), are both $\lambda_{1} \Delta t$, one has

$$
\begin{gathered}
\sum_{k_{2}=0}^{\infty} p\left(k_{1}, k_{2}\right)=p_{k_{1}}=e^{-\lambda_{1} \Delta t} \frac{\left(\lambda_{1} \Delta t\right)^{k_{1}}}{k_{1} !} \\
\operatorname{Var}_{1}=\left(\frac{\sigma_{01}}{2}\right)^{2}+\sigma_{1}^{2} \lambda_{1} \Delta t
\end{gathered}
$$

and similarly for $\operatorname{Var} R_{2}$.

Next, abbreviating $\mathbb{E}\left[R_{1} R_{2} \mid \Delta N_{t}=k_{1}, \Delta \tilde{N}_{t}=k_{2}\right]=\mathbb{E}\left[R_{1} R_{2} \mid k_{1}, k_{2}\right]$, we calculate,

$$
\begin{aligned}
\mathbb{E}\left[R_{1} R_{2}\right] & =\sum_{k_{1}, k_{2}=0}^{\infty} p\left(k_{1}, k_{2}\right) \mathbb{E}\left[R_{1} R_{2} \mid k_{1}, k_{2}\right] \\
& =\sum_{k_{1}, k_{2}=0}^{\infty} p\left(k_{1}, k_{2}\right) \int_{-\infty}^{\infty} \int_{1} x_{1} x_{2} f^{\left(k_{1}, k_{2}\right)}\left(x_{1}, x_{2}\right) d x_{1} d x_{2}
\end{aligned}
$$

where $f^{\left(k_{1}, k_{2}\right)}\left(x_{1}, x_{2}\right)$ is the joint density for $\hat{R}_{1}, \hat{R}_{2}$ defined in (10) The covariance can then be expressed by writing

$$
\begin{gathered}
\mathbb{E} R_{1} \mathbb{E} R_{2}=\mu_{01} \mu_{02}=\sum_{k_{1}, k_{2}=0}^{\infty} p\left(k_{1}, k_{2}\right) \mu_{01} \mu_{02} \\
\operatorname{Cov}\left(R_{1}, R_{2}\right)=\sum_{k_{1}, k_{2}=0}^{\infty} p\left(k_{1}, k_{2}\right)\left(\iint_{-\infty}^{\infty} x_{1} x_{2} f^{\left(k_{1}, k_{2}\right)}\left(x_{1}, x_{2}\right) d x_{1} d x_{2}-\mu_{01} \mu_{02}\right) .
\end{gathered}
$$

The term in the parentheses is $\operatorname{Cov}\left(\hat{R}_{1}\left(k_{1}\right), \hat{R}_{2}\left(k_{2}\right)\right)=\rho\left(k_{1}, k_{2}\right) \sigma_{R_{1}}\left(k_{1}\right) \sigma_{R_{2}}\left(k_{2}\right)$ (see (12) in Section 2) so we can write

$$
\operatorname{Cov}\left(R_{1}, R_{2}\right)=\sum_{k_{1}, k_{2}=0}^{\infty} p\left(k_{1}, k_{2}\right) \rho\left(k_{1}, k_{2}\right) \sigma_{R_{1}}\left(k_{1}\right) \sigma_{R_{2}}\left(k_{2}\right) .
$$

We can then write the overall correlation, $\rho_{T}$, as

$$
\begin{aligned}
\rho_{T} & :=\frac{\operatorname{Cov}\left(R_{1}, R_{2}\right)}{\sqrt{\operatorname{VarR_{1}} \sqrt{\operatorname{Var} R_{2}}}} \\
& =\frac{\sum_{k_{1}, k_{2}=0}^{\infty} p\left(k_{1}, k_{2}\right) \rho\left(k_{1}, k_{2}\right) \sigma_{R_{1}}\left(k_{1}\right) \sigma_{R_{2}}\left(k_{2}\right)}{\sqrt{\left(\frac{\sigma_{01}}{2}\right)^{2}+\sigma_{1}^{2} \lambda_{1} \Delta t} \sqrt{\left(\frac{\sigma_{02}}{2}\right)^{2}+\sigma_{2}^{2} \lambda_{2} \Delta t}} .
\end{aligned}
$$

In order to show that $\rho_{T}=1$ can be unity when the correlation between the bivariate Poisson processes, $\Delta N_{t}$ and $\Delta \tilde{N}_{t}$ is one, we note first the result below, which follows immediately from the definition, (44) . If the correlation between $\Delta N_{t}$ and $\Delta \tilde{N}_{t}$ approaches 1 then one must have $\lambda_{01} \rightarrow 0, \lambda_{02} \rightarrow 0$, while $\lambda_{12} \rightarrow 1$, so that

$$
\lim _{\lambda_{0 j} \rightarrow 0} p\left(k_{1}, k_{2}\right)=\left\{\begin{array}{ccc}
0 & \text { if } & k_{1} \neq k_{2} \\
e^{-\lambda_{12} \frac{\lambda_{11}^{k_{1}}}{k_{1} !}} & \text { if } & k_{1}=k_{2}
\end{array}\right.
$$

and thus, $p\left(k_{1}, k_{2}\right)$ reduces to $p\left(k_{1}, k_{2}\right)=p_{k_{1}} \delta\left(k_{1}, k_{2}\right)$ as the correlation approaches 1 .

In the limit as the correlation between $\Delta N_{t}$ and $\Delta \tilde{N}_{t}$ approaches 1 , the numerator of (51) has the form: 


$$
\begin{aligned}
& \sum_{k_{1}, k_{2}=0}^{\infty} p\left(k_{1}, k_{2}\right) \rho\left(k_{1}, k_{2}\right) \sigma_{R_{1}}\left(k_{1}\right) \sigma_{R_{2}}\left(k_{2}\right) \\
& =\sum_{k_{1}=0}^{\infty} p_{k_{1}} \rho\left(k_{1}, k_{1}\right) \sigma_{R_{1}}\left(k_{1}\right) \sigma_{R_{2}}\left(k_{1}\right) .
\end{aligned}
$$

If $\sigma_{01}=\sigma_{02}$ and $\sigma_{1}=\sigma_{2}$ (so the two types of variances for the supply and demand are equal), and $\rho\left(k_{1}, k_{2}\right)=1$ then the numerator reduces to

$$
\begin{aligned}
\sum_{k_{1}=0}^{\infty} p_{k_{1}} \sigma_{R_{1}}\left(k_{1}\right) \sigma_{R_{2}}\left(k_{1}\right) & =\sum_{k_{1}=0}^{\infty} p_{k_{1}}\left\{\left(\frac{\sigma_{01}}{2}\right)^{2}+\sigma_{1}^{2} k_{1} \Delta t\right\} \\
& =\left(\frac{\sigma_{01}}{2}\right)^{2}+\sigma_{1}^{2} \lambda_{1} \Delta t
\end{aligned}
$$

where we have have used the fact that the mean of $\Delta N_{t}$ is $\lambda_{1} \Delta t$, yielding the following result.

Theorem C.1. Suppose that $\sigma_{01}=\sigma_{02}$ and $\sigma_{1}=\sigma_{2}$, the correlation between $\Delta N_{t}$ and $\Delta \tilde{N}_{t}$ approaches 1 , and the covariance $\rho\left(k_{1}, k_{2}\right)$ is set as an arbitrary number $r \in(-1,1)$. Then $\rho_{T} \rightarrow r$.

From (51) it is clear -as one would expect - that one cannot attain the full range $(-1,1)$ of $\rho_{T}$ if the number of jumps in supply and demand are not correlated. Equation (51) can also be used to obtain the correlation when these two Poisson processes are independent. Using the standard result that independence implies

$$
p\left(k_{1}, k_{2}\right)=p_{k_{1}} p_{k_{2}}
$$

one has the correlation for the overall probability as

$$
\rho_{T}=\frac{\sum_{k_{1}, k_{2}=0}^{\infty} p_{k_{1}} p_{k_{2}} \rho\left(k_{1}, k_{2}\right) \sigma_{R_{1}}\left(k_{1}\right) \sigma_{R_{2}}\left(k_{2}\right)}{\sqrt{\left(\frac{\sigma_{01}}{2}\right)^{2}+\sigma_{1}^{2} \lambda_{1} \Delta t} \sqrt{\left(\frac{\sigma_{02}}{2}\right)^{2}+\sigma_{2}^{2} \lambda_{2} \Delta t}}
$$

Since $\sigma_{R_{1}}^{2}\left(k_{1}\right)=\left(\frac{\sigma_{01}}{2}\right)^{2}+k_{1} \sigma_{1}^{2}$ and likewise for $\sigma_{R_{2}}^{2}\left(k_{2}\right)$, one has the following exact total correlation under the condition that the Poisson processes are independent but the distribution of the jumps have covariance $\sigma\left(k_{1}, k_{2}\right)$ :

$$
\rho_{T}=\frac{\sum_{k_{1}, k_{2}=0}^{\infty} p_{k_{1}} p_{k_{2}} \sigma\left(k_{1}, k_{2}\right) \sqrt{\left(\frac{\sigma_{01}}{2}\right)^{2}+k_{1} \sigma_{1}^{2}} \sqrt{\left(\frac{\sigma_{02}}{2}\right)^{2}+k_{2} \sigma_{2}^{2}}}{\sqrt{\left(\frac{\sigma_{01}}{2}\right)^{2}+\sigma_{1}^{2} \lambda_{1} \Delta t} \sqrt{\left(\frac{\sigma_{02}}{2}\right)^{2}+\sigma_{2}^{2} \lambda_{2} \Delta t}} .
$$

Note that even if we set $\sigma_{01}=\sigma_{02}$ and $\sigma_{1}=\sigma_{2}$, and $\rho\left(k_{1}, k_{2}\right)=1$ we obtain

$$
\rho_{T}=\frac{\left(\sum_{k_{1}=0}^{\infty} p_{k_{1}} \sqrt{\left(\frac{\sigma_{01}}{2}\right)^{2}+k_{1} \sigma_{1}^{2}}\right)^{2}}{\left(\frac{\sigma_{01}}{2}\right)^{2}+\sigma_{1}^{2} \lambda_{1} \Delta t} .
$$

The denominator can be written as $\sum_{k_{1}=0}^{\infty} p_{k_{1}}\left\{\left(\frac{\sigma_{01}}{2}\right)^{2}+\sigma_{1}^{2} k_{1}\right\}$, so that the Cauchy-Schwarz inequality implies $\rho_{T} \leq 1$ with equality holding only for trivial Poisson probabilities $\left\{p_{k_{1}}\right\}$.

This shows that when the Poisson processes are uncorrelated the minimum of $\rho_{T}$ even with full anticorrelation, $\sigma\left(k_{1}, k_{2}\right)=-1$ will exceed -1 . However, when there is a bivariate Poisson process, the value of $\rho_{T}$ can span the range $(-1,1)$ depending on the $\sigma\left(k_{1}, k_{2}\right)$.

Appendix D. Randomness and the Central Limit Theorem in supply and demand. We consider a large number of agents buying an asset, and argue that the random orders satisfy the the Central Limit Theorem (CLT), and model the price change within a discrete formulation of the basic equation 


$$
P^{-1} \frac{d P}{d t}=\frac{\mathcal{D}(t, P(t, \Gamma), \omega)}{\mathcal{S}(t, P(t, \bar{\Gamma}), \bar{\omega})}-1
$$

We consider a discrete set of times, $\left\{t_{j}\right\}_{j=1}^{\infty}$, and prices, $\left\{P_{i}\right\}_{i=1}^{N}$. We let $\Gamma_{j}:=\left\{\omega_{0}, \ldots, \omega_{j}, \bar{\omega}_{0}, \ldots, \bar{\omega}_{j}\right\}$ where $\omega \in \Omega, \bar{\omega} \in \Omega$ are random values that will be chosen from a distribution. When the computed price is not exactly one of those values, we approximate with the closest of the $P_{i}$. The demand at the time $t_{j}$ can depend on the existing price, $P\left(t_{j}, \Gamma_{j}\right)$ and on $t_{j}$ directly, due to a multitude of factors. In addition, the demand will depend on a random value that depends on $\omega_{j}$. The situation is the same for supply. The randomness can depend on the time and price in a complicated way. As noted earlier, script $\mathcal{D}$, is used to indicate the full demand (including randomness) while $D$ is used for the deterministic part, or equivalently the expected value of demand (assuming the random term has vanishing mean). We will thus assume $\mathbb{E} \mathcal{D}=D$, and analogously for supply.

We illustrate the idea with a simple model below.

A basic model. One simple possibility is that the random variable, say, $R_{j}\left(\omega_{j}\right)$, alters the deterministic demand, $D(t, P(t, \Gamma))$ via

$$
\mathcal{D}\left(t_{j}, P\left(t_{j}, \Gamma_{j}\right), \omega_{j}\right)=D\left(t_{j-1}, P\left(t_{j-1}, \Gamma_{j-1}\right)\right)\left(1+R_{j}\left(\omega_{j}\right)\right),
$$

and analogously for supply with $\bar{R}_{j}\left(\bar{\omega}_{j}\right)$. In other words, at time $t_{j}$ we have a particular price, $P\left(t_{j-1}, \Gamma_{j-1}\right)$ that depends on the random variables chosen up through time $t_{j-1}$, i.e., $\Gamma_{j-1}$. If there were no additional randomness at time $t_{j}$ we would have the total demand, $\mathcal{D}$, that would lack the $R_{j}\left(\omega_{j}\right)$ term. Randomness introduced in this multiplicative form simply states that the magnitude of the randomness is proportional to the overall demand. For example, if the expectation of demand is a million units, we would expect that the standard deviation would be much larger than if it were a hundred units. Thus, simply adding $R_{j}$ to the demand would not be meaningful.

Next, we consider the distribution of $R_{j}$. For an actively traded stock, we can assume that there are many independent traders and investors that can be approximated by some distribution. If the number of such traders is sufficiently large, the distribution of the sum can be approximated well by the normal distribution. This is a consequence of the Central Limit Theorem (CLT) which is applied here directly to the large number of agents (individuals or institutions). Consider, for example, 1000 agents bidding on an asset at a particular time with a particular distribution that is not necessarily normal. For a broad set of distributions, the CLT will apply, and we can thus assume that the aggregate demand is normal. This is in sharp contrast to trying to apply CLT directly to prices, since prices are not chosed directly by agents, but arise from a complex process. As shown in the literature [14, [28] even the quotient of two normals is not normal, though in some regimes of parameters, e.g., near the mean, the distribution of the quotient can be approximated by a Gaussian distribution.

The price will depend on the set of $\omega_{i}, \bar{\omega}_{i}$ prior to that time, i.e., $\Gamma_{i}$. Let

$$
\Delta P\left(t_{j}, \Gamma_{j}\right):=P\left(t_{j}, \Gamma_{j}\right)-P\left(t_{j-1}, \Gamma_{j-1}\right) .
$$

The discrete version of the price equation can be written, with $R_{j+1}$ normal,

$$
\frac{\Delta P\left(t_{j+1}, \Gamma_{j+1}\right)}{P\left(t_{j}, \Gamma_{j}\right)}=\frac{D\left(t_{j}, P\left(t_{j}, \Gamma_{j}\right)\right)\left(1+R_{j+1}\left(\omega_{j+1}\right)\right)}{S\left(t_{j}, P\left(t_{j}, \Gamma_{j}\right)\right)\left(1+\bar{R}_{j+1}\left(\bar{\omega}_{j+1}\right)\right)}-1
$$

so that as we obtain the successive terms,

$$
P\left(t_{j+1}, \Gamma_{j+1}\right)=\frac{D\left(t_{j}, P\left(t_{j}, \Gamma_{j}\right)\right)\left(1+R_{j+1}\left(\omega_{j+1}\right)\right)}{S\left(t_{j}, P\left(t_{j}, \Gamma_{j}\right)\right)\left(1+\bar{R}_{j+1}\left(\bar{\omega}_{j+1}\right)\right)} P\left(t_{j}, \Gamma_{j}\right)
$$

We note that in this simple model, the randomness influences the demand at all prices uniformly through (53) .

A more general model. We now consider distributions that can depend not only on time, but on the price as well. For example, one might have greater variance for prices that are extreme. Changing notation, we focus on a single time $t_{j}$ and write the random variables $\mathcal{D}, \mathcal{S}$ and $X$ as

$$
\begin{aligned}
& \mathcal{D}=\left(\mathcal{D}_{1}, \ldots, \mathcal{D}_{N}\right), \quad \mathcal{S}=\left(\mathcal{S}_{1}, . ., \mathcal{S}_{N}\right), \\
& X=\left(X_{1}, . ., X_{2 N}\right)=\left(\mathcal{D}_{1}, \ldots, \mathcal{D}_{N}, \mathcal{S}_{1}, . ., \mathcal{S}_{N}\right),
\end{aligned}
$$


where $\mathcal{D}_{k}$ is the demand at price $P_{k}$. At this time $t_{j}$ there is a deterministic component (i.e., expected value) of the demand at each price $P_{k}$ with $k=1,2, . ., N$, denoted $\mu_{k}$ with $k=1,2, . ., N$. Similarly, for the supply, $X_{N+1}, \ldots X_{2 N}$ one has $\mu_{N+1}, . ., \mu_{2 N}$. The covariance matrix, $\Sigma$, is arbitrary, provided it is positive definite. Thus, one can specify the correlations between the supply and demand at different prices. Note that the correlation between the supply and demand at a given price will generally be negative, and close to -1 . Hence, the density of $X$ is a multivariate normal described by

$$
\begin{aligned}
f(x ; \mu, \Sigma) & =(2 \pi)^{-N}|\Sigma|^{-1 / 2} e^{-Q_{2 N}(x ; \mu, \Sigma) / 2} \\
Q_{2 N}(x ; \mu, \Sigma) & :=(x-\mu)^{T} \Sigma^{-1}(x-\mu) .
\end{aligned}
$$

The price change at time $t_{j+1}$ when the price $P\left(t_{j}, \Gamma_{j}\right)=P_{k}$ is thus given by

$$
\begin{aligned}
\frac{\Delta P\left(t_{j+1}, \Gamma_{j+1}\right)}{P\left(t_{j}, \Gamma_{j}\right)} & =\frac{\mathcal{D}_{k}\left(t_{j}\right)}{\mathcal{S}_{k}\left(t_{j}\right)}-1=\frac{X_{k}\left(t_{j}\right)}{X_{N+k}\left(t_{j}\right)}-1, \text { i.e., } \\
P\left(t_{j+1}, \Gamma_{j+1}\right) & =P\left(t_{j}, \Gamma_{j}\right) \frac{X_{k}\left(t_{j}\right)}{X_{N+k}\left(t_{j}\right)} .
\end{aligned}
$$

If we consider the full nonlinear model with general $G$ subject to the conditions stated earlier (including $\left.G(1)=0, G^{\prime}>0\right)$ the equation has the form

$$
P^{-1} \frac{d P}{d t}=G\left(\frac{\mathcal{D}(t, P(t, \Gamma), \omega)}{\mathcal{S}(t, P(t, \bar{\Gamma}), \bar{\omega})}\right) .
$$

The discrete model above can then be written as

$$
P\left(t_{j+1}, \Gamma_{j+1}\right)=\left[G\left(\frac{X_{k}\left(t_{j}\right)}{X_{N+k}\left(t_{j}\right)}\right)+1\right] P\left(t_{j}, \Gamma_{j}\right)
$$

Appendix E. Issue of negative supply or demand. We consider, for simplicity, the situation without the jump terms as the issues are similar.

(I) Does negative demand make any sense in finance? While a standard interpretation is that all buy orders are demand and all sell orders are supply, there is also the concept of selling short, which could be interpreted as negative demand, for example. Whether this is useful depends on the other equations coupled with the price equation, as in the asset flow equations. In other words, if there is a group of investors that is largely on the buy-side, it may be a useful mathematical concept to consider selling short as negative demand. Whether this is useful depends on the other equations coupled with the price equation, as in the asset flow equations.

For commodities, negative price means that one has to pay someone to take it away, as happened with oil prices in May 2020. This could be interpreted as negative net demand.

In the text, we consider the mathematics in full generality as the mathematical issue involving a quotient of such random variables in other contexts.

(II) The normal distribution is used in many measurable quantities that are positive by definition, e.g. IQ, prices, etc. While negative values are theoretically possible from the normal distribution, the standard deviation is sufficiently small compared to the mean that the probability of a negative value is infinitesimal. The standard equation for asset price, (1) , can lead to negative prices, for example. Indeed, letting $\mu=0$,(i.e., no trend) and $\sigma=$ const for simplicity, and fixing a time interval $\Delta t$, so $\Delta W \sim \mathcal{N}(0, \Delta t)$, one has

$$
\frac{\Delta P}{P} \tilde{=} \sigma \Delta W .
$$

Thus, one has

$$
P(t+\Delta t) \stackrel{=}{=}(t)(1+\sigma \Delta W)
$$


The probability of a negative price is the probability that a random variable $X \sim \mathcal{N}\left(0, \sigma^{2} \Delta t\right)$ is less than -1 , i.e., this is a $\left(\sigma(\Delta t)^{1 / 2}\right)^{-1}$ standard deviation event. For the S\&P 500, or a typical stock, the standard deviation of the price is about $0.5 \%$ or $1 \%$ of the price. Thus, we set $\Delta t=1$ and $\sigma=0.01$, so the equation above indicates (using 1\%) that the probability of going to negative price in one day is a 100 standard deviation event. Hence, this possibility is negligible within this framework.

The situation is similar for negative demand or supply, as one could verify - in principle - from the order book data. Also, this can be related to relative price change using the price change equation [6, and shown to be the same order of magnitude as the probability of negative prices.

(III) There is also the possibility of using conditional probability. This, of course, changes the distribution by an infinitesimal amount (provided the standard deviation is small compared to the mean), and it forces strictly positive values of supply and demand. We can think of this as a random choice of demand that is made from a normal distribution with mean $\mu_{\mathcal{D}}$ and variance $\sigma_{\mathcal{D}}^{2}$. If the value is positive, it is used. In the extremely rare event that it is negative, the choice is discarded. When $\mu_{\mathcal{D}} / \sigma_{\mathcal{D}}$ exceeds 5 or 6 , the two densities would be nearly identical for practical purposes. However, the analysis of this conditional probability is useful in other contexts beyond price, demand and supply, particularly, when the standard deviation is not small compared to the mean.

(IV) There are other ways to modify the normal distribution so that the random variable has no negative values, e.g., by truncation. The resulting distribution is of course not exactly normal, and in practice, the difference between this approach and the conditional approach we have used would be negligible in this application, but could be significant when the standard deviation is not small compared to the mean.

Acknowledgement. The author thanks the Hayek Fund for Scholars for its support.

\section{References}

[1] Aoki, M. and Yoshikawa, H., 2006. Reconstructing Macroeconomics: A Perspective from Statistical Physics and Combinatorial Stochastic Processes. Cambridge University Press.

[2] Bachelier L. 1900. Théorie de la spéculation. Ann. Sci. École Norm. Sup., 17, 21-86.

[3] Brock, W.A. and Hommes, C.H., 1998. Heterogeneous beliefs and routes to chaos in a simple asset pricing model. Journal of Economic Dynamics and Control, 22, 1235-1274.

[4] Bouchaud, J.P. and Potters, M., 2000. Theory of financial risks. From Statistical Physics to Risk Management. Cambridge University Press.

[5] Black, F., Scholes, M. 1973. The pricing of options and corporate liabilities. J. Political Economy, 81, 637-654.

[6] Caginalp, G., Balevonich, D. 1999. Asset flow and momentum: Deterministic and stochastic equations. Phil. Trans. Royal Soc. A., 357, 2119-2113.

[7] Caginalp, C. and Caginalp, G., 2018. The quotient of normal random variables and application to asset price fat tails. Physica A: Statistical Mechanics and its Applications 499, 457-471.

[8] Caginalp, C. and Caginalp, G., 2019. Price equations with symmetric supply/demand; implications for fat tails. Economics Letters, 176, 79-82.

[9] Caginalp, G. and Ermentrout, G.B., 1990. A kinetic thermodynamics approach to the psychology of fluctuations in financial markets. Applied Mathematics Letters, 3, 17-19.

[10] Carroll, C., 2006. The method of endogenous gridpoints for solving dynamic stochastic optimization problems. Economics Letters, 91, 312-320. 
[11] Champagnat, N., Deaconu M., Lejay A., Navet N. and Boukherouaa S. 2013. An empirical analysis of heavy-tails behavior of financial data: The case for power laws. HAL archives-ouvertes.

[12] Cont, R. and Tankov, P., 2004. Financial modeling with jump processes. Chapman \& Hall/CRC, London.

[13] Daníelsson, J., Jorgensen, B.N., Samorodnitsky, G., Sarma, M. and de Vries, C.G., 2013. Fat tails, VaR and subadditivity. Journal of Econometrics, 172, 283-291.

[14] Díaz-Francés, E. and Rubio, F. J. 2013. On the existence of a normal approximation to the distribution of the ratio of two independent normal random variables. Stat. Papers, 1-15.

[15] DeSantis, M. and Swigon, D., 2018. Slow-fast analysis of a multi-group asset flow model with implications for the dynamics of wealth. PloS one, 13, p.e0207764.

[16] Eom, C., Kaizoji, T., Kang, S.H. and Pichl, L., 2019. Bitcoin and investor sentiment: statistical characteristics and predictability. Physica A: Statistical Mechanics and its Applications, 514, 511-521.

[17] Eom, C., Kaizoji, T. and Scalas, E., 2019. Fat tails in financial return distributions revisited: Evidence from the Korean stock market. Physica A: Statistical Mechanics and its Applications, 526, p.121055.

[18] Erdelyi, A., 1956. Asymptotic Expansions. Dover Publications.

[19] Evans, L.C., 2010. Partial Differential Equations. 2nd Ed. Am. Math. Soc.

[20] Evans, G.W. and Honkapohja, S., 1998. Stochastic gradient learning in the cobweb model. Economics Letters, 61, 333-337.

[21] Fama, E. 1965. The Behavior of Stock-Market Prices. J. Business, 38, 34-105.

[22] Fouque, J.P., Papanicolaou, G., Sircar, R. and Sølna, K., 2011. Multiscale stochastic volatility for equity, interest rate, and credit derivatives. Cambridge University Press.

[23] Gabaix, X., 2016. Power laws in economics: An introduction. Journal of Economic Perspectives, 30, 185-206.

[24] Gabaix, X., Gopikrishnan, P., Plerou, V., and Stanley, H. E. 2006. Institutional investors and stock market volatility. The Quarterly Journal of Economics, 121, 461-504.

[25] Gjerstad, S. and Dickhaut, J., 1998. Price formation in double auctions. Games and economic behavior, 22, 1-29.

[26] Gjerstad, S., 2007. The competitive market paradox. Journal of Economic Dynamics and Control, 31, $1753-1780$.

[27] Gjerstad, S., 2013. Price dynamics in an exchange economy. Economic Theory, 52, 461-500.

[28] Geary, R. 1930. The frequency distribution of the quotient of two normal variates. J. Royal Stat. Soc. 93, 442-446.

[29] Gopikrishnan, P., Meyer, M., Amaral, L. and Stanley, H.E. 1998 Inverse cubic law for the distribution of stock price variations. Eur. Phys. J. B 3, 139-140. https://doi.org/10.1007/s100510050292

[30] Henderson, J. and Quandt, R. 1980. Microeconomic theory - A mathematical approach. McGraw-Hill, New York.

[31] Hirshleifer, J., Glazer, A. and Hirshleifer, D., 2005. Price theory and applications: decisions, markets, and information. Cambridge Univ. Press.

[32] Hull, J.C., 2015. Options, Futures and Other Derivatives, 9th Ed. Pearson.

[33] Jeitschko, T.D., 1999. Equilibrium price paths in sequential auctions with stochastic supply. Economics Letters, 64, 67-72. 
[34] Jansen, D.W. and De Vries, C.G., 1991. On the frequency of large stock returns: Putting booms and busts into perspective. The review of economics and statistics,18-24.

[35] Kawamura, K., 1973. The structure of bivariate Poisson distribution. Kodai Math Sem. Rep. 246-256.

[36] Kemp, M. 2011. Extreme events - robust portfolio construction in the presence of fat tails. Wiley Finance, Hoboken, NJ.

[37] Klenow, P.J. and Malin, B.A., 2010. Microeconomic evidence on price-setting. In Handbook of monetary economics (Vol. 3, pp. 231-284). Elsevier.

[38] Kirchler, M. and Huber, J., 2007. Fat tails and volatility clustering in experimental asset markets. Journal of Economic Dynamics and Control, 31, 1844-1874.

[39] Lux, T., 1996. The stable Paretian hypothesis and the frequency of large returns: an examination of major German stocks. Applied financial economics, 6,.463-475.

[40] LeBaron, B. and Samanta, R., 2005. Extreme value theory and fat tails in equity markets. Available at SSRN 873656.

[41] Mandelbrot, B. 1962. Sur certain prix speculatifs: faits empirique et modele base sur les processes stables additifs de Paul Levy. Comptes Rendus de l'Academie de Sciences, 254, 3968-3970.

[42] Mood, A.M., Graybill, F. A. and Boes, D.C., 1973 Introduction to the theory of statistics, 3rd Ed. McGraw-Hill.

[43] Mandelbrot, B. and Hudson, R. 2007. The misbehavior of markets: A fractal view of financial turbulence. Basic Books, New York.

[44] Marsaglia, G., 2006. Ratios of normal variables. J. of Stat. Soft 16, 1-10.

[45] H. Merdan, H. and Alisen, M., 2011. A mathematical model for asset pricing. App. Math. Comp., 218, 1449-1456.

[46] Milgrom, P., 2017. Discovering Prices: Auction Design in Markets with Complex Constraints. Columbia University Press.

[47] Mantegna, R.N. and Stanley, H.E., 1999. Introduction to econophysics: correlations and complexity in finance. Cambridge University press.

[48] Pham-Gia, T., Turkkan, N. and Marchand, E., 2006. Density of the ratio of two normal random variables and applications. Communications in Statistics-Theory and Methods, 35(9), pp.1569-1591.

[49] Plott, C., and Pogorelskiy, K. 2016. Call market experiments: efficiency and price discovery through multiple calls and emergent Newton adjustments. Available at SSRN: https://ssrn.com/abstract=2602034 or http://dx.doi.org/10.2139/ssrn.2602034.

[50] Porter, D., Rassenti, S., Roopnarine, A. and Smith, V., 2003. Combinatorial auction design. Proceedings of the National Academy of Sciences, 100, 11153-11157.

[51] Rachev, S.T. ed., 2003. Handbook of Heavy Tailed Distributions in Finance. Handbooks in Finance, Book 1. Elsevier.

[52] Raberto, M., Cincotti, S., Focardi, S.M. and Marchesi, M., 2001. Agent-based simulation of a financial market. Physica A: Statistical Mechanics and its Applications, 299, 319-327.

[53] Rachev, S.T., Menn, C. and Fabozzi, F.J., 2005. Fat-tailed and skewed asset return distributions: implications for risk management, portfolio selection, and option pricing. John Wiley \& Sons.

[54] Rachev, Z., Rocheva-Iotovo, B. and Stoyanov, S., 2010. Capturing fat tails. Risk, 23, p.72. 
[55] Rathgeber, A.W., Stadler, J. and Stöckl, S., 2019. Financial modelling applying multivariate Lévy processes: New insights into estimation and simulation. Physica A: Statistical Mechanics and its Applications, 532, p.121386.

[56] Si, Y., Nadarajah, S. and Song, X., 2020. On the distribution of quotient of random variables conditioned to the positive quadrant. Communications in Statistics-Theory and Methods, 49, 2514-2528.

[57] Taleb, N., Daniel, G. 2011. The problem is beyond psychology: The real world is more random than regression analyses. http://ssrn.com/abstract=1941792.

[58] Tong, Y. 1990. The multivariate normal distribution. Springer-Verlag, New York.

[59] Watson, D., Getz, M. 1981. Price theory and its uses, 5th Ed. University Press of America.

[60] Weintraub, E. R. 1979 Microfoundations, Cambridge University Press.

\section{CAPTION FOR FIGURE 1}

The graph of $y=r(x):=x-x^{-1}$ featuring the two branches is displayed. Calculations of the density for $r\left(R_{a}\right)$ required determining the intersection of a constant value of $y$ with $r(x)$. When $y>0$ the intersection in the upper half plane occurs at the values $x_{+}^{>}$for the right branch, and $x_{-}^{>}$for the left branch on the

$x$-axis. Analogously the intersections for the lower half plane $(y<0)$ occur at $x_{+}^{<}$and $x_{-}^{<}$. The qualitative features of $G_{\varepsilon}(x)$ are similar, and the same notation is used when $G_{\varepsilon}(x)$ replaces $r(x)$. 